\title{
Allgemeines Vertragsrecht und „Rechtsgeschäfts“-lehre im Draft Common Frame of Reference (DCFR)
}

\author{
von Prof. Dr. Phillip Hellwege, Augsburg
}

\section{Inhaltsübersicht}

I. Einleitung ..................................... 665

II. Allgemeine Vorschriften zu Verträgen und anderen „Rechtsgeschäften“ .. 666

III. Antidiskriminierung ........................... 670

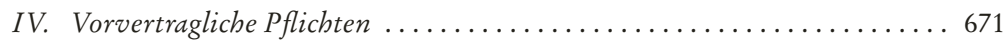

V. Vertragsschluss und Wirksamwerden von juridical acts ............ 673

VI. Verbraucherschützender Widerruf ........................6 674

VII. Unwirksamkeit ...................................676

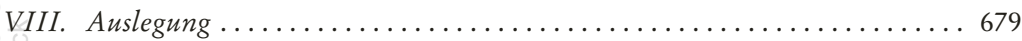

IX. Inhalt und Wirkungen von Verträgen ...................... 680

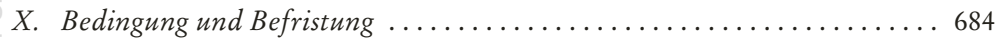

XI. Die Verallgemeinerung des allgemeinen Vertragsrechts ............. 684

XII. Der Definitionskatalog ............................ 686

XIII. Die vier Grundprinzipien ............................. 689

XIV. Die möglichen Funktionen des Draft Common Frame of Reference ..... 694

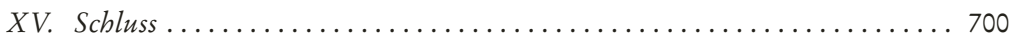

\section{Einleitung}

Seit Herbst 2009 liegt die vollständige Ausgabe des Draft Common Frame of Reference vor ${ }^{1}$. Ihr erster Band enthält im zweiten Buch allgemeine Bestimmungen über Verträge und andere juridical acts, und damit aus deutscher Sicht vor allem die Rechtsgeschäftslehre. Dieses zweite Buch soll in diesem Beitrag gewürdigt werden.

1 v. Bar/Clive (Hg.), Principles, Definitions and Model Rules of European Private Law. Draft Common Frame of Reference (DCFR), 6 Bd., München 2009. 


\section{Allgemeine Vorschriften zu Verträgen und anderen "Rechtsgeschäften“}

Der Titel des zweiten Buches: „Contracts and other juridical acts“, deutet bereits an, dass es vom Vertragsbegriff ausgeht und das allgemeine Vertragsrecht für andere juridical acts nur verallgemeinert. Der DCFR dreht so die Regelungstechnik des BGB um², das vom Allgemeinen zum Besonderen schreitet. Das zweite Buch des DCFR enthält damit genau genommen keine Rechtsgeschäftslehre, sondern nur eine allgemeine Vertragslehre. Diese Regelungstechnik findet sich schon in den Principles of European Contract Law (PECL). Freilich enthalten diese mit Art. 1:107 nur eine Vorschrift zur analogen Anwendung der Regeln zum allgemeinen Vertragsrecht auf andere Willensäußerungen:

„These Principles apply with appropriate modifications to agreements to modify or end a contract, to unilateral promises and to other statements and conduct indicating intention."

\section{Die Verfasser des DCFR sahen diese Regelungstechnik kritisch ${ }^{3}$ :}

„However, this technique is a short-cut which should only be used with great care and only when the appropriate modifications will be slight and fairly obvious. In this instance what modifications would be appropriate was not always apparent. It was therefore decided, as far back as 2004, to deal separately with other juridical acts."

Der DCFR entwickelt also die PECL fort, beschränkt sich aber nicht darauf juridical acts eigenständig zu regeln, sondern führt mit juridical act auch einen Begriff ein, den die PECL so nicht kennen. Vertrag und juridical act sind damit die Zentralbegriffe des zweiten Buches. Sie werden im einleitenden Kapitel („General provisions“) in Art. II.-1:101 definiert:

„(1) A contract is an agreement which is intended to give rise to a binding legal relationship or to have some other legal effect. It is a bilateral or multilateral juridical act.

(2) A juridical act is any statement or agreement, whether express or implied from conduct, which is intended to have legal effect as such. It may be unilateral, bilateral or multilateral.“

Der deutsche Leser wird juridical act als Rechtsgeschäft übersetzen wollen, und in der Tat wurde bereits behauptet (und bedauert), dass sich der DCFR hier (zu sehr) am deutschen Recht orientiere ${ }^{4}$. Doch sind beide Be-

${ }^{2}$ Kritisch hierzu Schmidt, Der ,juridical act“ im DCFR, ZEuP 18 (2010), 317.

3 v. Bar/Beale/Clive/Schulte-Nölke, Introduction, in: v. Bar/Clive (Fn. 1), S. 16.

${ }^{4}$ So Hesselink, The Common Frame of Reference as a Source of European Private Law, (2009) 83 Tulane LR 969. 
griffe nicht identisch ${ }^{5}$. Hieße es in Art. II.-1:101(2): „A juridical act is any statement, whether express or implied from conduct, which is intended to have legal effect“, dann würde es sich um eine Willenserklärung im deutschen Sinne handeln, wobei in der Definition fehlen würde, dass die Folge nur eintritt, weil sie gewollt ist ${ }^{6}$. Ein Rechtsgeschäft besteht zwar mindestens aus einer Willenserklärung, kann jedoch noch weitere Tatbestandsmerkmale enthalten, und die gewollte Rechtsfolge tritt erst ein, wenn der gesamte Tatbestand vorliegt $\mathrm{t}^{7}$. Solche weiteren Tatbestandsmerkmale sind in Art. II.-1:101(2) indes nicht vorgesehen. Im juridical act darf man aber auch nicht die deutsche Willenserklärung erkennen. Denn ein juridical act kann nach Art. II.-1:101(2) ebenfalls ein Vertrag sein. Ein Vertrag kommt nun aber durch zwei Willenserklärungen zustande. Wenden wir uns den rechtsvergleichenden notes $\mathrm{zu}^{8}$, so würden wir erwarten, dass sie über diese Unterschiede aufklären. Denn nur so würden die notes die in verschiedenen Rechten geprägten Juristen für diese Unterschiede sensibilisieren.

Sehr viel sorgfältiger arbeiten die Verfasser den Vertragsbegriff heraus. Er solle nur die Einigung, nicht aber das daraus resultierende Rechtsverhältnis bezeichnen. Schon die Autoren der Einleitung ${ }^{9}$ weisen auf dieses Begriffsverständnis hin: Der Vertrag werde geschlossen; er werde aber nicht erfüllt; erfüllt werde die aus dem Vertrag erwachsende Pflicht; nicht vom Vertrag werde zurückgetreten, sondern vom aus der Einigung resultierenden Verhältnis und den daraus erwachsenden Pflichten. Hier sehen die Verfasser der Einleitung einen Fortschritt gegenüber den PECL ${ }^{10}$ :

„A primary purpose of the DCFR is to try to develop clear and consistent concepts and terminology. In pursuit of this aim the Study Group gave much consideration to the most appropriate way of using terms like, contract' and ,obligation' [...]. One rea-

${ }^{5}$ A.A. Schmidt, ZEuP 18 (2010), $306 \mathrm{f}$. Nicht eindeutig Vaquer, Farewell to Windscheid?, 2009 ERPL 490 und 494.

${ }^{6}$ Vgl. Bork, Allgemeiner Teil des Bürgerlichen Gesetzbuchs, 3. Aufl. 2011, Rn. 395.

7 Larenz/Wolf, Allgemeiner Teil des Bürgerlichen Rechts, 9. Aufl. 2004, $\$ 22$ Rn. 10.

${ }^{8}$ Zum Aufbau in Regeltext, erläuternde comments und rechtsvergleichende notes vgl. v. Bar/Beale/Clive/Schulte-Nölke, Introduction, in: v. Bar/Clive (Fn. 1), S. 3.

${ }^{9}$ Der erste Band der vollständigen Ausgabe enthält eine von v. Bar/Clive/Beale/ Schulte-Nölke gezeichnete Introduction. Sie enthält einen Überblick über die Entstehung des DCFR, die Arbeit an ihm, dessen Zielsetzung, Inhalt, Aufbau und Struktur sowie Ausführungen zum Verhältnis des DCFR zu den PECL sowie zu den Arbeiten der Study Group on a European Civil Code und der Research Group on EC Private Law (Acquis Group).

10 v. Bar/Beale/Clive/Schulte-Nölke, Introduction, in: v. Bar/Clive (Fn. 1), S. 16. Vgl. aber die Kritik bei Jansen/Zimmermann, Vertragsschluss und Irrtum im europäischen Vertragsrecht, AcP 210 (2010), $207 \mathrm{ff}$. 
son for many of the drafting changes from the PECL is the clearer distinction now drawn $[\ldots]$ between a contract $[\ldots]$ and the relationship $[\ldots]$ to which it gives rise."

Doch hält das zweite Buch diese Begrifflichkeiten schon im einleitenden Kapitel nicht durch. Zwei Artikel später in Art. II.-1:103(1) heißt es:

"A valid contract is binding on the parties.“

Wieso ist der Vertrag als Einigung bindend? In der Terminologie des DCFR müsste die Bindung an das aus dem Vertrag resultierende Verhältnis und an die daraus folgenden Pflichten statuiert werden.

Der DCFR erklärt, anders als das deutsche Recht, nicht nur Verträge für bindend: Nach $\$ 311$ Abs. 1 BGB ist zur Begründung eines rechtsgeschäftlichen Schuldverhältnisses grundsätzlich ein Vertrag erforderlich. Einseitige Versprechen binden nicht. Eine Ausnahme enthält etwa für die Auslobung \657 BGB. Der DCFR geht in Art. II.-1:103(2) hierüber hinaus:

„A valid unilateral undertaking is binding on the person giving it if it is intended to be legally binding without acceptance."

Einseitige Versprechen sind also auch ohne Annahme durch den Versprechensempfänger bindend, wenn der Versprechende gebunden sein will. Der DCFR folgt damit einem Trend, der sich bereits in Art. 2:107 PECL niederschlug ${ }^{11}$. Damit mussten die Autoren des DCFR bei Formulierung des allgemeinen Vertragsrechts zwei Dinge im Auge behalten: es muss, soweit erforderlich, für andere juridical acts verallgemeinerbar sein, und es muss für einseitige Versprechen, bei denen es sich auch um unilateral juridical acts handelt, passen. Ob den Verfassern dies gelungen ist, darauf wird unten nach einer Würdigung der Einzelabschnitte zurückzukommen sein ${ }^{12}$.

Bereits in den allgemeinen Vorschriften zu Verträgen und anderen juridical acts finden wir in Art. II.-1:106(1) die Formfreiheit normiert. Anders als $\$ 125$ S. 1 BGB bestimmen die allgemeinen Vorschriften des DCFR keine Rechtsfolge bei Verstoß gegen eine besonders angeordnete Form. Doch setzt Art. II.-1:106(2) diese Rechtsfolge voraus: Ist ein Vertrag oder anderer juridical act formunwirksam, so kann unter Umständen ein Anspruch auf Ersatz des Vertrauensschadens begründet sein:

„Where a contract or other juridical act is invalid only by reason of non-compliance with a particular requirement as to form, one party (the first party) is liable for any loss suffered by the other (second party) by acting in the mistaken, but reasonable, belief that it was valid if the first party: (a) knew it was invalid; (b) knew or could reasonably be expected to know that the second party was acting to that party's potential

${ }^{11}$ Vgl. hierzu statt aller Zimmermann, Vertrag und Versprechen, in: Festschrift für Andreas Heldrich, 2005, S. $467 \mathrm{ff}$.

12 Siehe unten Gliederungspunkt XI. 
prejudice in the mistaken belief that it was valid; and (c) contrary to good faith and fair dealing, allowed the second party to continue so acting."

Anders als Art.II.-1:106(1) findet Abs. 2 keine Vorgänger in den PECL, Principles of the Existing EC Contract Law (ACQP) oder Unidroit-Principles of International Commercial Contracts 2004 (PICC). Und das deutsche Recht geht hier weiter: Ein Verstoß gegen Treu und Glauben kann ausnahmsweise dazu führen, dass ein formnichtiges Rechtsgeschäft als gültig behandelt wird ${ }^{13}$. Freilich darf ein deutscher Jurist an einer bewusst anders getroffenen Entscheidung im DCFR keinen Anstoß nehmen, wenn sie überzeugend präsentiert wird. Doch genau hier liegt das Problem: Es ist zweifelhaft, ob mit Art. II.-1:106(2) solche Fälle befriedigend gelöst werden können, die im deutschen Recht der Anlass für die Entwicklung dieser Ausnahmen waren. Der Ersatz eines Vertrauensschadens wurde hier gerade nicht als ausreichend angesehen ${ }^{14}$. Der comment zu Art. II.-1:106(2) paraphrasiert die Regel freilich nur, ohne andere Problemlösungen zu erwähnen und rechtsvergleichende notes fehlen insoweit völlig.

Art. II.-1:107 behandelt gemischte Verträge. Die ACQP, PECL und PICC (2004) kennen keine vergleichbare Regel. Art. II.-1:107(2) geht von der im deutschen Recht sogenannten Kombinationstheorie aus ${ }^{15}$, ist freilich so offen formuliert, dass mit ihm auch jede andere Lösung erzielt werden kann. Die Regel ist also unschädlich. Dennoch ist sie nicht unproblematisch: Die rechtsvergleichenden notes offenbaren, dass kaum ein europäisches Recht gemischte Verträge gesetzlich regelt. Die Lösung wird wohl den Gerichten überlassen. In den notes hätten die Autoren also die mitgliedstaatlichen Rechtsprechungen analysieren müssen. Das fehlt. Es werden nur zwei deutsche Entscheidungen angeführt. Die gefundene Regel kann daher kaum für sich in Anspruch nehmen, auf Grundlage eines breiten Rechtsvergleichs geschaffen worden zu sein. Zudem hätten sich die Verfasser die Frage stellen müssen, ob das Fehlen einer entsprechenden Regel in den meisten Rechten nicht darauf hindeutet, dass die Problemlösung überhaupt besser den Gerichten überlassen wird und eine gesetzliche Regelung unterbleiben sollte. Auch diese Frage findet keine Erörterung.

Weiterhin finden wir im ersten Kapitel des zweiten Buches in Art. II.-1:108 das Problem der Teilunwirksamkeit geregelt:

„Where only part of a contract [...] is invalid or ineffective, the remaining part continues in effect if it can reasonably be maintained without the invalid or ineffective part."

13 Statt aller Palandt/Ellenberger, 70. Aufl. 2011, \125 Rn. $22 \mathrm{ff.}$

14 Vgl. nur BGH (Urteil v. 5.2.1957), BGHZ 23, 249.

15 Statt aller MK-BGB/Emmerich, 5. Aufl. 2007, \311 Rn. 46. 
Prima facie überzeugt diese Vorschrift, geht sie doch von einer Restgültigkeit aus und folgt so nicht $\$ 139$ BGB, sondern dem im Rechtsvergleich vorherrschenden Prinzip. Dass die notes den Eindruck erwecken, auch $\$ 139$ BGB statuiere das Prinzip der Restgültigkeit ${ }^{16}$, sei nur am Rande bemerkt ${ }^{17}$. Wie schon zuvor Art. 4:116 PECL und Art. 3.16 PICC (2004) für die Teilanfechtung kommt es entscheidend auf die reasonableness an. Zur Anwendung von Art. II.-1:108 heißt es in den comments ${ }^{18}$ :

"Circumstances which might be taken into account in assessing the reasonableness of upholding the remaining part include [...] whether the parties would have agreed to a contract consisting only of the remaining parts of the contract [...]."

Den Parteiwillen zu berücksichtigen, ist sinnvoll und findet Bestätigung im Rechtsvergleich. Nur ist seine Berücksichtigung in Art. II.-1:108 nicht möglich, wird doch der Begriff reasonableness in Art. I.-1:104 ganz bewusst rein objektiv gefasst:

„Reasonbleness is to be objectively ascertained, having regard to the nature and purpose of what is being done, to the circumstances of the case and to any relevant usages and practices."

Der Parteiwille ist nun aber kein objektives Kriterium. Obwohl reasonableness ein Zentralbegriff des DCFR ist ${ }^{19}$, wird er nicht einheitlich verwendet bzw. seine Definition wurde nicht mit allen Artikeln, in denen er vorkommt, abgestimmt.

\section{Antidiskriminierung}

Das zweite Kapitel des zweiten Buches widmet sich der Antidiskriminierung (non-discrimination). Den PECL und die PICC (2004) fehlt ein entsprechender Abschnitt, und vom dritten Kapitel der ACQP weichen die Vorschriften des DCFR ab, basieren aber ebenso wie letztere vor allem auf den Richtlinien 2000/43/EG und 2004/113/EG ${ }^{20}$. Niemand darf wegen des Geschlechts, der ethnischen Herkunft oder Rasse diskriminiert werden: Art. II.-

16 v. Bar/Clive (Fn. 1), Note 1 zu Art. II.-1:108 (S. 159).

17 Vgl. zum deutschen Recht nur MK-BGB/Busche, 5. Aufl. 2006, \139 Rn. 1.

18 v. Bar/Clive (Fn. 1), Comment zu Art. II.-1:108 (S. 158).

19 Kritisch zur Häufigkeit dieses Begriffes Pasa, The DCFR, the ACQP and the Reactions of Italian Legal Scholars, (2010) 18 ERPL 234.

20 RiL 2000/43/EG zur Anwendung des Gleichbehandlungsgrundsatzes ohne Unterschied der Rasse oder der ethnischen Herkunft; RiL 2004/113/EG zur Verwirklichung des Grundsatzes der Gleichbehandlung von Männern und Frauen beim Zugang zu und bei der Versorgung mit Gütern und Dienstleistungen. 
2:101 DCFR ${ }^{21}$. Dieser Schutz existiert: „,in relation to a contract or other juridical act the object of which is to provide access to, or supply, goods, other assets or services which are available to the public". In Art. II.-2:102 folgt eine Definition des Begriffs discrimination, und Art.II.-2:103 führt eine Ausnahme ein:

„Unequal treatment which is justified by a legitimate aim does not amount to discrimination if the means used to achieve that aim are appropriate and necessary."

Der erzielte Schutz geht weiter als im deutschen Recht. So kennt der DCFR nicht den abgestuften Schutz des $\$ 19$ AGG bei Verträgen über die Vermietung von Wohnraum ${ }^{22}$. Und die comments zu Art. II.-2:103 suggerieren, dass auch die öffentliche Anzeige, ein privates WG-Zimmer zu vermieten, bereits unter die Wendung ,the object of which is to provide access to [...] assets [...] which are available to the public“ in Art. II.-2:101 fällt ${ }^{23}$ : Die Vermietung nur an Frauen durch eine weibliche Vermieterin könne nach Art. II.2:103 gerechtfertigt sein, andere Diskriminierungen dagegen nicht. Bereits die Arbeiten am AGG waren von zum Teil heftiger Kritik begleitet worden ${ }^{24}$, und so bleibt abzuwarten, ob der im Vergleich zum deutschen Recht weitergehende Schutz im DCFR nicht ebenfalls auf Ablehnung stoßen wird. Zumindest muss sich diese Fortentwicklung einer rechtspolitischen Diskussion stellen.

\section{Vorvertragliche Pflichten}

Das dritte Kapitel behandelt in fünf Abschnitten vorvertragliche Pflichten (marketing and pre-contractual duties). Der erste Abschnitt zu vorvertraglichen Informationspflichten ist in der Literatur bereits auf Kritik gestoßen ${ }^{25}$ : Er sei rechtspolitisch verfehlt, finde in den PECL kein Vorbild sowie im Ver-

21 Vgl. hierzu bereits Ebert/Pinkel, Restricting Freedom of Contract through Non-Discrimination Provisions?, (2009) 10 German LJ 1417 ff.; Thüsing/Horler, NonDiscrimination in the DCFR, in: Wagner (Hg.), The Common Frame of Reference: A View from Law \& Economics, 2009, S. 217 ff.; Leible, Non-Discrimination, in: Schulze (Hg.), Common Frame of Reference and Existing EC Contract Law, 2. Aufl. 2009, S. $125 \mathrm{ff}$.

22 Freilich ist das deutsche Recht insoweit nicht unproblematisch, vgl. nur MKBGB/Thüsing, 5. Aufl. 2007, Vor $\ 19$ AGG Rn. 9 ff.

23 v. Bar/Clive (Fn. 1), Comment C zu Art. II.-2:103 (S. 185).

$24 \mathrm{Vgl}$. nur die Übersicht bei MK-BGB/Thüsing, 5. Aufl. 2007, Einl. AGG Rn. $20 \mathrm{ff}$.

25 Mit Blick auf die Interim Outline Edition Whittaker, The ,Draft Common Frame of Reference', 2008, S. 101 ff.; Eidenmüller/Faust/Grigoleit/Jansen/Wagner/ Zimmermann, Der Gemeinsame Referenzrahmen, JZ 2008, 544 f.; Herresthal, Con- 
gleich und Unionsprivatrecht keine Stütze, sondern basiere auf wenig überzeugenden Verallgemeinerungen des Unionsprivatrechts und übersetze dessen lauterkeitsrechtliche Bestimmungen ins Vertragsrecht. Damit hätten die Verfasser ihren Auftrag überschritten. Und das Ergebnis kann handwerklich nicht überzeugen. So enthält der Abschnitt Begrifflichkeiten aus dem Lauterkeitsrecht. Man hätte erwartet, dass die Verfasser bei einer Übertragung lauterkeitsrechtlicher Bestimmungen ins Vertragsrecht auch eine vertragsrechtliche Terminologie finden.

Zudem wurde das Zusammenspiel mit anderen Normkomplexen nicht beachtet: Wirbt ein Unternehmer öffentlich, muss er den Verbraucher nach Art. II.-3:102(2) über „peculiarities related to payment, delivery, performance and complaint handling, if they depart from the requirements of professional diligence" bereits in der Werbung informieren. Andernfalls kommt der Vertrag nach Art. II.-3:109(2) so zustande, wie es der Verbraucher ohne diese Informationen erwarten durfte. Die in Art. II.-3:102(2) geforderten Informationen finden sich meist in AGB. In welchem Verhältnis stehen diese Vorschriften also zum AGB-Recht? Der Unternehmer muss über solche „peculiarities“ informieren, die von den "requirements of professional diligence“ abweichen. Muss er also nur über unangemessene oder überraschende Klauseln informieren ${ }^{26}$ ? Lässt Art. II.-3:109(2) den Umkehrschluss zu, dass der Unternehmer darauf verweisen kann, er habe in seinen Werbeaussagen genügend auf seine AGB hingewiesen, und er müsse daher nicht mehr die Einbeziehungsvoraussetzungen des Art. II.-9:103 erfüllen? Hat der Unternehmer gegen seine Informationspflicht aus Art. II.-3:102(2) verstoßen, folgt dann aus Art. II.-3:109(2), dass der Unternehmer entsprechende AGB überhaupt nicht mehr zum Vertragsinhalt machen kann, selbst wenn er die Voraussetzungen des Art. II.-9:103 erfüllt? In ihrer vorliegenden Fassung sind diese Regeln also allenfalls ein erster, sehr gewagter Vorschlag.

Der zweite Abschnitt enthält zwei Artikel zur Pflicht des Unternehmers im elektronischen Geschäftsverkehr, Vorrichtungen, die Eingabefehler vermeidbar machen, vorzuhalten, und zur Pflicht des Unternehmers, den Erhalt eines Angebots oder einer Annahme im elektronischen Geschäftsverkehr zu bestätigen. Im dritten Abschnitt folgen zwei Artikel zur Pflicht, Verhandlungen im Einklang mit good faith and fair dealing zu führen, und zur Pflicht, vertrauliche Informationen, die der Verhandlungspartner im Rahmen von Vertragsverhandlungen offenbart hat, nicht an Dritte weiterzugeben oder anderweitig

sumer Law in the DCFR, in: Wagner (Fn. 21), S.178. Die Outline Edition positiv bewertend Twigg-Flesner, Pre-Contractual Duties, in: Schulze (Fn. 21), S. 95 ff.

26 In diese Richtung geht wohl der Vorschlag von Orlando, The Use of Unfair Contractual Terms as an Unfair Commercial Practice, ERCL 2011, $25 \mathrm{ff.}$ 
zu verwenden, und im vierten Abschnitt findet sich eine Vorschrift zu unbestellten Leistungen. Diese Vorschriften scheinen gelungen. Nur Art. II.-3:401 ist verwirrend gefasst: Art. II.-3:401(1)(b) normiert, dass bei unbestellten Leistungen auch nicht vertragliche Ansprüche ausgeschlossen seien, und die comments machen deutlich, dass davon insbesondere bereicherungsrechtliche Ansprüche erfasst seien ${ }^{27}$. Doch heißt es dann in Art. II.-3:401(2)(b), dass dies nicht gelte, wenn die Leistungen erbracht worden seien „in error or in such other circumstances that there is a right to reversal of an unjustified enrichment". Also sind bereicherungsrechtliche Ansprüche doch nicht ausgeschlossen? Im letzten Abschnitt normiert Art. II.-3:501(1) schließlich:

„Where any rule in this Chapter makes a person liable for loss caused to another person by breach of a duty, the other person has a right to damages for that loss.“

Die Anspruchsgrundlagen finden sich in den Regeln, auf die diese Norm verweist. Die Aussage von Art. II.-3:501(1) ist damit, dass eine Partei schadensersatzpflichtig ist, wenn sie schadensersatzpflichtig ist.

\section{Vertragsschluss und Wirksamwerden von juridical acts}

Das vierte Kapitel (formation) regelt in drei Abschnitten den Vertragsschluss und das Wirksamwerden von juridical acts. Es beginnt im ersten Abschnitt mit allgemeinen Regeln. Art. II.-4:101 normiert die Voraussetzungen des Vertragsschlusses. Die Parteien müssen sich rechtlich binden wollen und eine Einigung erzielt haben. Aus deutscher Sicht klingt dies nach einer Selbstverständlichkeit. Die Bedeutung dieser Vorschrift liegt vor allem in dem Halbsatz „without any further requirements“: Voraussetzungen, wie etwa die consideration des englischen Rechts, werden nicht aufgestellt ${ }^{28}$. Die Frage, ob eine Partei sich rechtlich binden will, wird ähnlich wie im deutschen Recht nach Art. II.-4:102 beurteilt „from the party's statements or conduct as they were reasonably understood by the other party". Freilich verzichtet der DCFR, ebenso wie schon zuvor Art. 2:102 PECL, auf die Voraussetzung des deutschen Rechts, dass dieses Verständnis dem Handelnden zurechenbar sein muss ${ }^{29}$.

Der zweite Abschnitt enthält elf Artikel zu Angebot und Annahme und der dritte Abschnitt drei Artikel zu juridical acts. Hier werden die Regeln zum Vertragsschluss vor allem modifiziert, dass sie für unilateral juridical

27 v. Bar/Clive (Fn. 1), Comment G zu Art. II.-3:401 (S. 260).

28 v. Bar/Clive (Fn. 1), Comment E zu Art. II.-4:101 (S. 265).

${ }^{29}$ Hierzu Medicus, Allgemeiner Teil des BGB, 10. Aufl. 2010, Rn. 326. 
acts passen. Aus deutscher Sicht sind beide Abschnitte im Großen und Ganzen gelungen ${ }^{30}$. Sie enthalten zwar Abweichungen vom deutschen Recht. So ist nach Art. II.-4:208(1) eine modifizierte Annahme nur dann eine Ablehnung, wenn die Modifikation des Angebots wesentlich ist. Und eine verspätete Annahme kann der Empfänger als wirksam behandeln, muss dies aber dem Annehmenden unverzüglich mitteilen: Art. II.-4:207(1). Doch wird dies rechtsvergleichend überzeugend hergeleitet.

Drei Dinge müssen aber kritisch angemerkt werden: Zum einen überzeugt der Aufbau dieses Kapitels nicht. Die Kapitelüberschrift ist mit formation so weit gefasst, dass sie den Vertragsschluss und das Wirksamwerden von juridical acts umfasst. Doch die Bestimmungen des ersten Abschnitts beziehen sich sodann allein auf Verträge und nicht auf sonstige juridical acts. Es handelt sich damit nicht, wie die Abschnittsüberschrift: „General provisions“, glauben macht, um allgemeine Vorschriften. Die Autoren des DCFR haben die ersten beiden Abschnitte dieses vierten Kapitels auf dem zweiten Kapitel der PECL basiert und dabei übersehen, dass die Ergänzung eines dritten Abschnitts zu anderen juridical acts eine Umstrukturierung des gesamten Kapitels erfordert hätte. Zum anderen erweckt die Überschrift des dritten Abschnitts („Other juridical acts“) den Eindruck, das Wirksamwerden von juridical acts werde umfassend behandelt. Es wird aber nur das Wirksamwerden von unilateral juridical acts geregelt. Nach Art. II.-1:101(2) gibt es jedoch auch multilateral juridical acts. Ihr Zustandekommen und Wirksamwerden wird nicht behandelt. Schließlich kommt es zu einer unschönen Doppelung ${ }^{31}$ : Die comments zu Art. II.-1:101 machen deutlich, dass es sich auch bei Angebot und Annahme um juridical acts handelt ${ }^{32}$. Dass die Annahme mit Zugang wirksam wird, regelt Art. II.-4:205(1). Das gleiche regelt sodann allgemein Art. II.-4:301(c).

\section{Verbraucherschützender Widerruf}

Das fünfte Kapitel widmet sich dem verbraucherschützenden Widerruf (right to withdrawal). Der erste Abschnitt regelt die Ausübung und Folgen des Widerrufs. Der zweite Abschnitt enthält zwei Widerrufsrechte. Art. II.5:201 normiert dabei ein Widerrufsrecht bei Verträgen, die außerhalb von Geschäftsräumen geschlossen worden sind. Dessen Abs. 1 lautet:

$30 \mathrm{Vgl}$. aber die detaillierte Kritik bei Jansen/Zimmermann, AcP 210 (2010), $209 \mathrm{ff}$. Zu Art. II.-4:209 Lord Mance, The Common Frame of Reference, ZEuP 18 (2010), 460.

31 So auch die Kritik von Vaquer, 2009 ERPL $493 \mathrm{f}$.

32 v. Bar/Clive (Fn. 1), Comment B zu Art. II.-1:101 (S. 125). 
„A consumer is entitled to withdraw from a contract under which a business supplies goods, other assets or services [...] to the consumer [...], if the consumer's offer or acceptance was expressed away from the business premises."

Art. II.-5:201 soll, wie schon Art. 5:A-01 ACQP, das Widerrufsrecht bei Haustürgeschäften und das bei Fernabsatzverträgen in sich vereinen. Die Idee einer solchen Zusammenführung ist auf den ersten Blick bestechend, doch bei näherem Hinsehen überwiegen die Bedenken ${ }^{33}$ : Das Widerrufsrecht bei Haustürgeschäften basiert auf der Überrumpelungsgefahr, das bei Fernabsatzverträgen auf der Informationsasymmetrie. Zwei Widerrufsrechte, die auf unterschiedliche Ausprägungen der Unterlegenheit des Verbrauchers reagieren, derart zu vereinen, verdeckt diese Unterschiede und erschwert damit eine teleologische Auslegung.

Zudem führt die vorgelegte Verallgemeinerung zu Anwendungsproblemen. Nehmen wir ein alltägliches Beispiel: In der Breiten Straße in Köln baut die Obsthändlerin O täglich ihren Obststand auf. Der Verbraucher V kauft Obst. Das Widerrufsrecht der Richtlinie 85/577/EWG ${ }^{34}$ ist ebensowenig einschlägig wie das der Richtlinie 97/7/EG ${ }^{35}$. Die Anwendung des Art. II:-5:201 bereitet dagegen Schwierigkeiten. Kann man einen Verkaufsstand im öffentlichen Raum als business premise bezeichnen? Wohl kaum. Denn dann hätte man auch bei Zeitungsständen, von denen aus Passanten aggressiv angesprochen werden, um ein Abonnement abzuschließen, kein Widerrufsrecht. In dieser Situation soll es nach dem Willen der Verfasser, anders als nach der Richtlinie 85/577/EWG, aber in Übereinstimmung mit $₫ 312$ Abs. 1 S. 1 Nr. 3 $\mathrm{BGB}^{36}$, jedoch ein Widerrufsrecht geben ${ }^{37}$. Nach der vorliegenden Fassung hätte jeder Kunde der Obsthändlerin ein Widerrufsrecht. Schließlich fällt auf, dass eine mit $₫ 312$ Abs. 3 Nr. 1 BGB vergleichbare Vorschrift fehlt. Freilich haben die Verfasser auf eine entsprechende Regel bewusst verzichtet ${ }^{38}$.

33 Vgl. Eidenmüller/Faust/Grigoleit/Jansen/Wagner/Zimmermann, JZ 2008, 545 f.; Herresthal (Fn. 25), S. 176; Eidenmüller, Why Withdrawal Rights?, ERCL 2011, $1 \mathrm{ff}$.

34 RiL 85/577/EWG betreffend den Verbraucherschutz im Falle von außerhalb von Geschäftsräumen geschlossenen Verträgen.

35 RiL 97/7/EG über den Verbraucherschutz bei Vertragsabschlüssen im Fernabsatz.

36 \312 BGB geht insoweit über die RiL 85/577/EWG hinaus: Staudinger/Thüsing, Neubearbeitung 2005, $\$ 312$ Rn. 111.

37 v. Bar/Clive (Fn. 1), Comment B zu Art. II.-5:201 (S. 388).

38 v. Bar/Clive (Fn. 1), Comment I zu Art. II.-5:201 (S. 394). 


\section{Unwirksamkeit}

Im siebten Kapitel werden Unwirksamkeitsgründe (grounds of invalidity) behandelt. Im ersten Abschnitt finden sich Bestimmungen zum Anwendungsbereich des Kapitels und zur anfänglichen Unmöglichkeit. Ausdrücklich wird im gesamten Kapitel nur die Unwirksamkeit von Verträgen geregelt, Art. II.-7:101(3) erklärt diese Bestimmungen indes „with any necessary adaptations" auf alle juridical acts für anwendbar. Der zweite Abschnitt regelt Willensmängel, und der letzte Abschnitt ist mit „Infringement of fundamental principles or mandatory rules“ überschrieben und erfasst Fälle des $\$ 134$ BGB, aber auch solche des $\$ 138$ Abs. 1 BGB.

Die Voraussetzungen eines beachtlichen Irrtums in Art. II.-7:201 basieren im Wesentlichen auf Art. 4:103 PECL, wobei noch ergänzt wurde, dass ein Vertrag auch anfechtbar ist, wenn eine Partei durch Verstoß gegen vorvertragliche Informationspflichten einen Irrtum verursacht hat ${ }^{39}$. Wie schon Art. 4:103 PECL weicht auch Art. II.-7:201 vom deutschen Recht ab: Ein Vertrag ist nur bei einem erkannten oder fahrlässig unerkannten, kausalen Rechts- oder Tatsachenirrtum anfechtbar, wenn dieser verursacht, ausgenutzt oder geteilt war. Ausgeschlossen ist die Anfechtung bei einem unentschuldbaren Irrtum und wenn der Irrende das Irrtumsrisiko übernommen hatte oder es ihm aus anderen Gründen zugeordnet werden muss. Einem Irrtum gleichgestellt ist eine „inaccuracy in the expression or transmission“: Art. II.-7:202. Von dieser Regelung sind wohl die aus dem deutschen Recht bekannten Erklärungs- und Übermittlungsirrtümer erfasst ${ }^{40}$. Wie schon zuvor Art. 4:105 PECL normiert auch Art. II.-7:203, daß die Irrtumsanfechtung ausgeschlossen ist, wenn die andere Partei den Irrenden an seinem wirklichen Willen festhalten will. Art. II.-7:204 enthält einen Anspruch auf Ersatz des Vertrauensschadens. Es handelt sich nicht um einen Anspruch gegen den Anfechtenden, sondern um einen Anspruch desjenigen, der sich bei Vertragsschluss auf falsche Informationen der anderen Partei verlassen hat. Dieser Anspruch besteht selbst, wenn der Geschädigte kein Anfechtungsrecht hat. Er scheint daher falsch verortet zu sein. Er hätte in das dritte Kapitel gehört. Anfechtungsgründe sind weiter die arglistige Täuschung (fraud), Zwang und Drohung (coercion or threats) und unbillige Ausnutzung (unfair exploitation). Im Folgenden finden sich noch Regeln zur Ausübung der Anfechtung, ihrer Frist,

39 Eine umfassende Würdigung des Irrtumsrechts findet sich bei Jansen/Zimmermann, AcP 210 (2010), 229 ff., 240 ff.; und mit Blick auf die Interim Outline Edition bei Eidenmüller/Faust/Grigoleit/Jansen/Wagner/Zimmermann, JZ 2008, 546 f.

40 Kritisch hierzu Jansen/Zimmermann, AcP 210 (2010), 246. 
ihren Folgen, zur teilweisen Anfechtung, zur Abdingbarkeit des Anfechtungsrechts und zum Verhältnis zu anderen Rechtsbehelfen.

Das siebte Kapitel regelt nur Unwirksamkeitsgründe. Die verschiedenen Unwirksamkeitsgrade werden hier dagegen nicht erklärt, ihre Definition vielmehr vorausgesetzt, doch finden wir entsprechende Begriffserläuterungen in einer dem Regeltext vorangestellten Liste. Hier sind die Begriffe avoidance, ineffective, invalid, void und voidable erklärt ${ }^{41}$ :

„Invalid' in relation to a juridical act or legal relationship means that the act or relationship is void or has been avoided."

„Valid' [...] means that the act or relationship is not void and has not been avoided.“

Void und voidable beschreiben also die zwei Formen der invalidity:

„Void' [...] means that the act or relationship is automatically of no effect from the beginning."

„Voidable $[\ldots]$ means that the act or relationship is subject to a defect which renders it liable to be avoided and hence rendered retrospectively of no effect."

Avoidance wird entsprechend definiert:

„Avoidance $[\ldots]$ is the process whereby a party or, as the case may be, a court invokes a ground of invalidity so as to make the act or relationship, which has been valid until that point, retrospectively ineffective from the beginning."

Und schließlich:

„Ineffective in relation to a contract or other juridical act means having no effect, whether that state of affairs is temporary or permanent, general or restricted.“

Prima facie sind diese Definitionen gelungen. Doch ist es unvorteilhaft Begriffe von mehreren Seiten her zu fassen. So macht es der DCFR aber für den Begriff void. Er wird nicht nur von der Wirkung her gefasst („of no effect“), sondern auch von der Art her, wie die Wirkung geltend gemacht werden muss: im Fall der voidness muss die Wirkung überhaupt nicht geltend gemacht werden („automatically“). Diese Art der voidness wird gemeinhin mit dem $\mathrm{Zu}$ satz absolute versehen. Der Begriff der voidness kennt in allen europäischen Rechten Unterformen. Solche Unterformen sind dem DCFR unbekannt. Das hat eine sprachliche Merkwürdigkeit zur Folge. Das Verb to avoid bedeutet to make void. Man würde also meinen, die Wirkung der avoidance sei, dass der juridical act nunmehr void ist. So führt die Anfechtung im deutschen Recht ja auch dazu, dass das Rechtsgeschäft als von Anfang nichtig anzusehen ist. Ähnlich halten es andere europäische Rechte. Doch den Begriff void darf man beim DCFR nicht verwenden, um die Folge einer avoidance zu bezeichnen, tritt diese Rechtsfolge doch nicht automatisch ein. Daher sprechen die Defi-

41 v. Bar/Clive (Fn. 1), S. 65-82. 
nitionen der Begriffe voidable und avoidance auch davon, dass der Vertrag ineffective wird.

Der Begriff ineffective und die Paare valid/invalid und void/voidable stehen auf verschiedenen Abstraktionsebenen. Invalid ist ein Oberbegriff zu void und voidable. Darüber steht der Begriff ineffective. Die mittlere wird mit der unteren Abstraktionsebene definiert: „Invalid' [...] means that the act $[\ldots]$ is void or has been avoided." Diese Definition blickt nach unten. Bei der unteren Abstraktionsebene springt die Begriffserklärung dann nach ganz oben, indem der Begriff effective verwendet wird: „Void', [...] means that the act $[\ldots]$ is automatically of no effect from the beginning." Hier finden wir nun eine zirkuläre Definition: „,Ineffective“ [...] means having no effect [...].“ Das allein ist nicht besonders schön.

Hinzu kommt, dass der Gegenstand der Unwirksamkeit auf den unterschiedlichen Abstraktionsebenen wechselt: ineffective sind Verträge und andere juridical acts. Die übrigen Unwirksamkeitsgrade beziehen sich dagegen auf juridical acts und legal relationships. Die Autoren des DCFR hatten indes versprochen, dass sie Vertrag und das daraus resultierende Verhältnis strikt trennen, und sie sehen hierin gerade einen Fortschritt des DCFR gegenüber den PECL ${ }^{42}$. Dieses Versprechen haben die Autoren damit nicht eingelöst: Man fragt sich, was es bedeutet, dass sich voidness auch auf eine legal relationship bezieht und dass voidness über den Begriff ineffectiveness definiert wird, dann aber eine legal relationship als Gegenstand einer ineffectiveness überhaupt nicht vorgesehen ist. Und man fragt sich, ob es gewollt ist, dass sich die ineffectiveness allein auf juridical acts und Verträge bezieht, dass sich aber die übrigen Unwirksamkeitsgrade auch auf das aus einem Vertrag resultierende Verhältnis beziehen können.

Die Autoren waren sich zudem nicht bewusst, dass diese Begriffe auf drei Abstraktionsebenen angesiedelt sind. Denn sie verwenden in der Definition von avoidance die Begriffe valid und ineffective gleichrangig nebeneinander. „,Avoidance $[\ldots]$ is the process [...] to make the act [...], which has been valid [...] ineffective [...].“ Diese unsaubere Verwendung beider Begriffe resultiert auch daraus, dass der Definitionskatalog mit der invalidity nur eine Form der ineffectiveness nennt, obwohl es mehrere Formen geben muss. Der DCFR kennt in Art. III.-1:106 auflösende Bedingungen:

„Upon fulfilment of a resolutive condition, the relevant right, obligation or relationship comes to an end.“

${ }^{42}$ Siehe oben das Zitat zu Fn. 10. 
Das Recht kann nach Bedingungseintritt nicht als void bezeichnet werden, weil die in der Definition geforderte ex tunc-Wirkung fehlt. Es ist nicht voidable, weil die Wirkung der auflösenden Bedingung automatisch eintritt. Das Recht ist nach Bedingungseintritt auch nicht invalid, weil von invalidity nur gesprochen wird, wenn etwas void oder avoided ist. Das Recht ist weiterhin valid! Das kann nicht richtig sein und deutet auf eine verfehlte Begriffsbildung hin. Aber das Recht muss ineffective sein. Der DCFR kennt also in der Tat mehrere Formen der ineffectiveness. Dass die Definitionen mit der invalidity dennoch nur eine Form der ineffectiveness benennen, führt dazu, dass die Begriffe austauschbar verwendet werden, obwohl sie dies nicht sind.

In der Gesamtschau haben die Autoren des DCFR die Unwirksamkeitsbegriffe nicht durchdrungen. Das überrascht nicht, handelt es sich doch um eine Materie, die vergleichend kaum aufgearbeitet ist ${ }^{43}$. Dass es sich allenfalls um Begriffsannäherung handelt, zeigt sich auch am Folgenden. Schon im deutschen Recht werden die Begriffe der Nichtigkeit und der Anfechtung nur im Bürgerlichen Recht (nahezu) einheitlich verwendet. Geht man darüber hinaus, werden sie anders gebraucht, so im Prozess- (Nichtigkeitsklage) oder im Insolvenzrecht (Insolvenzanfechtung). Im acquis setzt sich diese Tendenz fort. So finden wir in der Verordnung 1346/2000 über Insolvenzverfahren ${ }^{44}$ die Begriffe voidness und voidability. Sie werden hier aber nicht im Sinne der Definitionen des DCFR verwendet. Die vom DCFR angestrebte einheitliche Terminologie ist von vornherein also beschränkt. Und nicht einmal der DCFR hält die angestrebte einheitliche Begrifflichkeit durch. So spricht Art. II.-1:106(2) bei Formverstoß von einer invalidity. Aber meint er damit im Sinne der Definition, dass der Vertrag nichtig oder anfechtbar ist? Auch Art.IV.H.-2:101 bezeichnet eine Schenkung, die nicht formwirksam ist, als nicht valid. Ist die Schenkung also vielleicht nur anfechtbar? Dagegen spricht Art. IV.G.-4:104 ausdrücklich von void, um die Folgen eines Formverstoßes zu bezeichnen.

\section{Auslegung}

Im ersten Abschnitt des achten Kapitels zur Vertragsauslegung finden wir im Kern die Regelungen der PECL, diesmal mit wenigen Ergänzungen. Hinzugefügt wurde auch der zweite Abschnitt zur Auslegung anderer juridical acts. Art. II.-8:201 tritt dabei an die Stelle von Art. II.-8:101 und modifiziert

43 Eine erste Annäherung versucht Hellwege, Unwirksamkeit, in: Basedow/Hopt/ Zimmermann (Hg.), Handwörterbuch des Europäischen Privatrechts, 2009, S. 1582 ff.

44 VO EG/1346/2000 über Insolvenzverfahren. 
ihn leicht. Auffälligste Änderung ist, dass es auf die übereinstimmenden Parteiwillen nicht ankommt. Das ist eine Selbstverständlichkeit, die auch über Art. II.-8:202 hätte erzielt werden können:

„The provisions of Section I, apart from its first Article, apply with appropriate adaptations to the interpretation of a juridical act other than a contract."

Und auch sonst enthält Art. II.-8:101 nur begriffliche Anpassungen, die zudem unachtsam vorgenommen wurden: Art. II.-8:201(3)(b) wurde aus Art. II.-8:101(3)(b) kopiert, ohne den Begriff contract durch den des juridical act $\mathrm{zu}$ ersetzen.

\section{Inhalt und Wirkungen von Verträgen}

Das neunte Kapitel widmet sich in vier Abschnitten dem Inhalt und den Wirkungen von Verträgen (contents and effects of contracts). Hier haben die Verfasser nur noch zum Teil auf die PECL zurückgegriffen. Der erste Abschnitt wendet sich dem Vertragsinhalt zu. Wir finden Vorschriften, die aus deutscher Sicht zur Auslegung und zum Vertragsschluss gehören, die aber freilich auch schon in den PECL an dieser Stelle verortet waren: Der Vertragsinhalt könne sich aus einer ausdrücklichen oder konkludenten Einigung, aus gesetzlichen Regeln, Geschäfts- oder Handelsgebräuchen ergeben: Art. II.9:101(1). Eine, in deutscher Terminologie, ergänzende Auslegung dürfe erfolgen, wenn dabei Natur und Zweck des Vertrages, die den Vertragsschluss begleitenden Umstände und der Grundsatz des good faith and fair dealing berücksichtigt werden: Art. II.-9:101(2)-(4).

Aufmerksamkeit verdient Art. II.-9:102: Vorvertragliche Äußerungen einer Partei können Vertragsinhalt werden. Die Verfasser erklären, dass sich die mit dieser Regel erreichten Ergebnisse in der Regel auch im Wege der Auslegung erzielen ließen. Die Regel ist also überflüssig, aber unschädlich. Die einzig bedeutsame Klarstellung findet sich in Abs.4: Werbeaussagen eines Herstellers oder eines Vorverkäufers können auch einem Unternehmer zugerechnet werden. Abs. 4 verallgemeinert den Gedanken, der sich im deutschen Recht in $\$ 434$ Abs. 1 S. 3 BGB findet. Abs. 4 ist noch aus einem weiteren Grund interessant: Die deutsche Lehre betont im Rahmen der Auslegung, dass einer Erklärung nur dann der Inhalt, wie er sich aus Sicht des Empfängers nach Treu und Glauben ergibt, beigemessen werden kann, wenn dieser Inhalt dem Erklärenden zurechenbar ist ${ }^{45}$. Dieser Zurechnungsgedanke fin-

45 Siehe nur Medicus (Fn. 29), Rn. 326. 
det sich weder in Art. II.-4:10246 noch in Art. II.-8:101(3). Art. II.-9:102(4) lässt er sich indes entnehmen: Die Werbeaussage des Herstellers oder des Vorverkäufers wird nicht Vertragsinhalt, wenn der Unternehmer sie nicht kannte oder kennen konnte. Es stellt sich daher die Frage, ob dieser Gedanke verallgemeinert werden darf.

Weiter fällt auf, dass die Frage nach dem Vertragsinhalt im DCFR an verschiedenen Stellen behandelt wird: Trifft eine Partei eine vorvertragliche Informationspflicht und hat sie gegen diese verstoßen, kommt der Vertrag mit dem Inhalt zustande, „as the other party has reasonably expected as a consequence of the absence or incorrectness of the information“: Art. II.-3:109(2). Den umgekehrten Fall, dass die Partei vorvertragliche Informationen gibt, regelt Art. II.-9:102. Schließlich finden sich die allgemeinen Auslegungsregeln. Ob es klug ist, dieses einheitliche Problem auseinanderzureißen, wird sich erweisen müssen. So wird es auf Art. II.-9:102 wohl nur ankommen, wenn sich die vorvertragliche Information als fehlerhaft herausstellt und die Partei, die diese Information gegeben hat, sich nicht an sie gebunden fühlt. Besteht aber zugleich eine vorvertragliche Informationspflicht nach Art. II.-3:101 ff. und hat diese Partei gegen diese Pflicht verstoßen, indem sie fehlerhafte Informationen erteilt hat, so ist nicht (oder nicht nur?) Art. II.-9:102, sondern (oder sōndern auch?) Art. II.-3:109(2) anwendbar. Das ist insoweit von Bedeutung, als beide Vorschriften unterschiedlich an das Problem herangehen: Nach Art. II.-3:109(2) muss sich der Unternehmer an seiner fehlerhaften Information festhalten lassen, wenn der Verbraucher reasonably von einem entsprechenden Vertragsinhalt ausgehen durfte. Art. II.-9:102(2) formuliert es umgekehrt: Der Unternehmer muss sich an seiner fehlerhaften Information festhalten lassen, außer der Verbraucher musste reasonably davon ausgehen, dass er sich auf diese Information nicht verlassen durfte. Beide Normen sind damit nicht optimal aufeinander abgestimmt.

Art. II.-9:103 regelt die Einbeziehung nicht individuell ausgehandelter Vertragsklauseln. Er entspricht dem deutschen $\$ 305$ Abs. 2 BGB, ist aber nicht auf AGB beschränkt. Anders als im deutschen Recht und in den Acquis Principles sind Einbeziehungsproblematik und Inhaltskontrolle nicht gemeinsam, sondern getrennt geregelt. Anders als die PECL behandelt der DCFR die Einbeziehung nicht im Kapitel zum Vertragsschluss, sondern in dem zum Vertragsinhalt. Die Verfasser des DCFR folgen damit der Systematik der englischen Literatur ${ }^{47}$. Auch inhaltlich orientiert sich Art. II.-9:103(1) weitgehend an der englischen Literatur ${ }^{48}$ : Nicht individuell ausgehandelte Klau-

46 Siehe oben den Text zu Fn. 29.

47 Kritisch Pfeiffer, Non-Negotiated Terms, in: Schulze (Fn. 21), S. 184.

$48 \mathrm{Zu}$ den Unterschieden etwa Whittaker (Fn. 25), S. 125 f. 
seln werden Vertragsinhalt, wenn der Verwender „took reasonable steps to draw the other party's attention to them, before or when the contract was concluded“. Die comments verdeutlichen, dass dafür in der Regel zweierlei erforderlich ist: Der Verwender muss die andere Partei auf die Klauseln hinweisen und die andere Partei muss die Möglichkeit haben, sich über ihren Inhalt $\mathrm{zu}$ informieren ${ }^{49}$. Diese Voraussetzungen kommen in $\$ 305$ Abs. 2 BGB aber deutlicher zum Ausdruck: Warum haben sich die Verfasser bei Fassung von Art. II.-9:103(1) also nicht am deutschen Recht orientiert? In den notes wird noch auf das Problem überraschender Klauseln Bezug genommen ${ }^{50}$. Eine ausdrückliche Regelung fehlt freilich, und auch in den comments wird nicht erläutert, ob das Problem über Art. II.-9:103(1) gelöst werden soll. Man könnte ja daran denken, dass bei solchen Klauseln an die reasonable steps des Verwenders strengere Anforderungen zu stellen sind. Der Rechtsvergleich hätte die Verfasser noch auf zwei weitere Fragen bringen können ${ }^{51}$ : Ist eine gesetzliche Regelung der Einbeziehungsvoraussetzungen überhaupt notwendig? In vielen europäischen Rechten werden die Voraussetzungen des Art. II.-9:103(1) ohne Probleme aus den allgemeinen Vertragsschlussregeln hergeleitet. Gilt dies vielleicht auch für den DCFR? Die Normierung dieser Voraussetzungen hätte dann allein klarstellende Funktion. Aber diese Funktion erfüllt Art. II.9:103(1) nur unzureichend, weil er die beiden Voraussetzungen, Hinweis und Kenntnisnahmemöglichkeit, nicht klar zum Ausdruck bringt. Zudem stellt sich die Frage nach der richtigen Rechtsfolge bei Nichterfüllung der Voraussetzungen. Art. II.-9:103(1) spricht wie Art. 2:104(1) PECL davon, dass sich der Verwender nur gegenüber der anderen Vertragspartei auf die Klauseln berufen kann („may be invoked against the other party only“), wenn dessen Voraussetzungen erfüllt sind. Die comments führen dazu aus ${ }^{52}$ :

„Terms which have been duly brought to the attention of a party will become part of the contract. If a party has not taken appropriate steps to bring the terms to the other party's attention the contract is treated as having been made without the terms, if the party wishes this result."

Es soll also im Belieben der anderen Partei stehen, ob sie die Nichteinbeziehung geltend macht. Das passt nicht zum dogmatischen Rahmen der Einbeziehungsproblematik ${ }^{53}$.

49 v. Bar/Clive (Fn. 1), Comment D zu Art. II.-9:103 (S. 589 f.).

50 v. Bar/Clive (Fn. 1), Comment B zu Art. II.-5:201 (S. 594).

$51 \mathrm{Vgl}$. zum Folgenden Hellwege, Allgemeine Geschäftsbedingungen, einseitig gestellte Vertragsbedingungen und die allgemeine Rechtsgeschäftslehre, 2010, S. $349 \mathrm{ff}$.

52 v. Bar/Clive (Fn. 1), Comment G zu Art. II.-9:103 (S. 591).

53 Vgl. hierzu Hellwege (Fn. 51), S. 593 ff. 
Das neunte Kapitel enthält im vierten Abschnitt weiterhin Bestimmungen zur Inhaltskontrolle. Er basiert auf der Klauselrichtlinie ${ }^{54}$, geht indes auch über sie hinaus, als er nicht nur die Kontrolle missbräuchlicher Klauseln in Verbraucherverträgen erfasst, sondern drei Generalklauseln der Inhaltskontrolle enthält ${ }^{55}$ : Art. II.-9:403 betrifft Verbraucherverträge und erstreckt die Kontrolle auf alle nicht individuell ausgehandelten Vertragsklauseln, wobei ein Klammerzusatz im Regeltext andeutet, dass noch unklar ist, ob nicht sogar alle, auch individuell ausgehandelte, Klauseln von ihr erfasst sein sollen ${ }^{56}$. Eine solche Klausel ist missbräuchlich (unfair), wenn sie „significantly disadvantages the consumer, contrary to good faith and fair dealing“. In Verträgen zwischen Nichtunternehmern werden nur AGB auf ihren Inhalt kontrolliert: Art. II.-9:404. Der Kontrollmaßstab ist identisch formuliert wie in Art. II.-9:403. Die comments machen aber deutlich, dass der Kontrollmaßstab unterschiedlich ausfallen $\mathrm{kann}^{57}$. Das hätte sich im Regeltext widerspiegeln dürfen. Art. II.-9:405 enthält eine Generalklausel für den Unternehmerverkehr. Auch hier sind Kontrollgegenstand nur Vertragsbedingungen in AGB. Nun ist der Kontrollmaßstab ein anderer: Eine Klausel ist unangemessen, „if it is [...] of such a nature that its use grossly deviates from good commercial practice, contrary to good faith and fair dealing". In Deutschland wird diese Differenzierung ihre Befürworter wie auch ihre Gegner finden ${ }^{58}$, ist doch die Frage der AGB-Kontrolle im Unternehmerverkehr umstritten. Freilich weist die Lösung des DCFR noch handwerkliche Mängel auf: So findet sich keine Inhaltskontrolle von Klauseln in Verträgen zwischen einem Nichtunternehmer, der kein Verbraucher ist, also etwa einem eingetragenen Idealverein, und einem Unternehmer. Art. II.-9:403-405 sind tatbestandlich nicht einschlägig. Folgt daraus, dass ein solcher Vertrag überhaupt nicht der Inhaltskontrolle unterfällt? Das ist kaum vorstellbar. Zudem überzeugt nicht, dass eine Klausel im Unternehmerverkehr schon dann unangemessen sein soll, wenn sie „grossly deviates from good commercial practice, contrary to good faith and fair dealing“. Denn in dieser Formel findet sich nicht die Voraussetzung einer Benachteiligung des Vertragspartners. Eine Klausel kann also missbräuchlich sein, wenn sie vom good commercial practice grob abweicht, obwohl diese Ab-

54 RiL 93/13/EWG über missbräuchliche Klauseln in Verbraucherverträgen.

55 Vgl. bereits Willett, Unfair Terms, in: Antoniolli/Fiorentini (Hg.), A Factual Assessment of the Draft Common Frame of Reference, 2011, S. $53 \mathrm{ff}$.; Antoniolli/Fiorentini/Gordley, A Case-Based Assessment of the Draft Common Frame of Reference, (2010) 58 AJCL $348 \mathrm{ff}$. $538 \mathrm{f}$.

56 Kritisch Eidenmüller/Faust/Grigoleit/Jansen/Wagner/Zimmermann, JZ 2008,

57 v. Bar/Clive (Fn. 1), Comment C zu Art. II.-9:404 (S. 640).

58 Kritisch bereits Pfeiffer (Fn. 47), 185. 
weichung nicht den Vertragspartner benachteiligt. Können also Klauseln, die Dritte entgegen good commercial practice belasten, im Unternehmerverkehr als unangemessen gelten ${ }^{59}$ ? Das würde nicht zur Rechtsfolge der Unangemessenheit nach Art. II.-9:408 passen:

"A term which is unfair [...] is not binding on the party who did not supply it."

Eine unangemessene Klausel ist also nicht absolut, sondern nur relativ unwirksam. Aber warum soll die andere Partei sich auf eine Unangemessenheit berufen, wenn sie durch die Klausel nicht benachteiligt wird?

\section{$X$. Bedingung und Befristung}

Gleicht man den Inhalt des zweiten Buches des DCFR mit dem Allgemeinen Teil des BGB ab, so stößt man auf eine Lücke: Es fehlen Regeln zu Bedingung und Befristung, wie wir sie aus $\mathbb{S} \mathbb{S} 158 \mathrm{ff}$. BGB kennen. Doch verzichtet auch der DCFR nicht auf ihre Normierung. Nur erscheinen Bedingung und Befristung nicht im zweiten Buch mit dem Titel „Contracts and other juridical acts“, sondern im dritten Buch mit dem Titel „Obligations and corresponding rights" und damit in dem Buch, das aus deutscher Sicht das allgemeine Schuldrecht enthält. Diese Verortung ist nicht gelungen. Die Verfasser werden entgegnen, dass Bedingung und Befristung nicht den Vertrag berühren, sondern nur das daraus erwachsene Verhältnis, eben die Rechte und Pflichten ${ }^{60}$. Und das dritte Buch behandelt Pflichten und korrespondierende Rechte. Doch klärt Art. II.-1:101(1) darüber auf, dass ein Vertrag als Rechtsfolge nicht nur ein Rechtsverhältnis mit Pflichten und Rechten, sondern auch andere Rechtsfolgen haben kann. Auch bei solchen Verträgen sollten Bedingungen und Befristungen möglich sein.

\section{Die Verallgemeinerung des allgemeinen Vertragsrechts}

Nachdem wir uns einen Überblick über das zweite Buch des DCFR verschafft haben, ist es möglich, die eingangs aufgezeigte Fortentwicklung der PECL, andere juridical acts nicht nur im Wege der Verweisung, sondern eigenständig zu regeln, zu würdigen. Die Verfasser des DCFR führen zu

59 Kritisch zu diesem Kontrollmaßstab auch Mangeri, Is the DCFR ready to be adopted a s an Optional Instrument?, ERCL 2011, $219 \mathrm{ff}$. S. 16 .

${ }^{60}$ Vgl. v. Bar/Beale/Clive/Schulte-Nölke, Introduction, in: v. Bar/Clive (Fn. 1), 
Art. 1:107 PECL, wonach die Regeln der allgemeinen Vertragslehre entsprechend auch für andere Willensäußerungen gelten, aus ${ }^{61}$ :

„However, this technique is a short-cut which should only be used with great care and only when the appropriate modifications will be slight and fairly obvious. In this instance what modifications would be appropriate was not always apparent. It was therefore decided, as far back as 2004 , to deal separately with other juridical acts."

Dieses Versprechen haben die Verfasser des DCFR nicht eingelöst ${ }^{62}$ : Viele Regeln sind für Verträge formuliert, und es findet sich die Bestimmung, dass sie auch auf andere juridical acts Anwendung finden: „with any necessary adaptations“, so Art. II.-1:104(3) und II.-7:101(3). Wie diese „necessary adaptations" aussehen sollen, wird nicht deutlich. In anderen Vorschriften wird der Regeltext nur so modifiziert, dass neben dem Vertrag noch other juridical acts genannt werden, ohne dass Modifikationen nötig wären, so in Art. II.1:106, II.-1:108 oder II.-2:101. Hier ist mit ihrer Nennung wenig gewonnen. Weiter erweckt diese Regelungstechnik den Eindruck, dass dort, wo other juridical acts nicht genannt werden, die Regel auf diese auch keine Anwendung findet. Aber warum soll es bei einseitigen Versprechen, die nach DCFR bindend sind, keine vor-„,vertraglichen“ Pflichten geben? Dennoch finden Art. II.-3:101 ff. keine Anwendung. Ihre Anwendung kann auch bei anderen juridical acts geboten sein. Warum muss der Unternehmer im elektronischen Geschäftsverkehr nach Art. II.-3:201 nur bei dem Vertragsschluss technische Vorkehrungen treffen, die einen input-error auf Seiten des Verbrauchers verhindern? Kündigt der Verbraucher das Vertragsverhältnis, die Kündigung ist ein einseitiger juridical act, kann sich das gleiche Problem ergeben. Warum muss ein Unternehmer nach Art. II.-3:202 im elektronischen Geschäftsverkehr nur den Eingang eines Angebots oder einer Annahme bestätigen? Auch hier hat der Verbraucher bei Kündigungen ein gleichgelagertes Interesse an einer Bestätigung. Warum ist das gesamte neunte Kapitel auf Verträge beschränkt? Gibt es hier wirklich keinen Bedarf für eine Anwendung dieser Regeln auf andere juridical acts? Gerade weil der DCFR einseitige Versprechen nach Art. II.-1:103(2) generell als bindend anerkennt, liegt die Frage auf der Hand, ob nicht die Vorschriften des neunten Kapitels verallgemeinert werden müssen. Nur in zwei Fällen finden wir umfassende Regelungen zu anderen juridical acts: in Art. II.-4:301 ff. und Art. II.-8:201 f. Aber diese Regelungen konnten aus anderen Gründen nicht überzeugen ${ }^{63}$.

Zudem ist die prominente Stellung, die der DCFR anderen juridical acts zuschreibt, bereits aus grundlegenderer Sicht kritisiert worden. Martijn Hes-

61 v. Bar/Beale/Clive/Schulte-Nölke, Introduction, in: v. Bar/Clive (Fn. 1), S. 16.

62 So auch die Einschätzung von Schmidt, ZEuP 18 (2010), $318 \mathrm{f}$.

63 Siehe oben Gliederungspunkte V und VIII. 
selink meint, sie „makes the draft look more [...] Germanic than necessary“64, und Nils Jansen und Reinhard Zimmermann merken an, dass sie kaum auf dem acquis commun basiere ${ }^{65}$.

Schließlich wurde laut Einleitung über diese Weichenstellung bereits 2004 entschieden ${ }^{66}$. Doch die Arbeiten am DCFR begannen erst $2005^{67}$ ! Das mit der Erarbeitung des DCFR beauftragte Netzwerk kann zu diesem Zeitpunkt noch nicht entschieden haben! Wer hat diese Entscheidung dann getroffen? War dies die Acquis Group? Die Acquis Principles folgen zwar nicht dem gleichen Ansatz, aber behandeln doch die gleiche Materie. Aber warum hat die Acquis Group dann endgültig über eine solch wichtige systematische Frage entscheiden können? Wegen der unterschiedlichen Zielsetzungen beider Gruppen hätten doch die von der Acquis Group getroffenen Entscheidungen nicht unbesehen übernommen werden dürfen. Und, so die Autoren der Einleitung, die Acquis Principles waren nur eine Grundlage für die Arbeiten am DCFR $^{68}$. Die Einleitung erweckt aber den Eindruck, als habe die Entscheidung nicht mehr zur Disposition gestanden. Einem Außenstehenden stellt sich die Frage, wie die Entscheidungsprozesse innerhalb des Netzwerkes abliefen: Es fehlt die gebotene Transparenz ${ }^{69}$.

\section{Der Definitionskatalog}

Eine Würdigung des zweiten Buchs des DCFR wäre unvollständig, wenn nicht der den Regeln vorangestellte Definitionskatalog berücksichtigt wird ${ }^{70}$, soweit er für das allgemeine Vertragsrecht des zweiten Buches relevant ist. Die Ausarbeitung eines solchen Katalogs gehörte zum Auftrag der Verfasser des DCFR $^{71}$. Die Definitionen werden über Art. I.-1:108(1) zum Bestandteil des

${ }^{64}$ Hesselink, (2009) 83 Tulane LR 969.

${ }^{65}$ Jansen/Zimmermann, AcP 210 (2010), 203. Kritisch auch Whittaker (Fn. 25), S. 6; Schmidt, ZEuP 18 (210), $310 \mathrm{ff}$.

${ }_{66}$ Siehe das Zitat oben zu Fn. 61.

${ }^{67} \mathrm{Vgl}$. v. Bar/Beale/Clive/Schulte-Nölke, Introduction, in: v. Bar/Clive (Fn. 1), S. 2 .

68 v. Bar/Beale/Clive/Schulte-Nölke, Introduction, in: v. Bar/Clive (Fn. 1), S. 18 f.

${ }_{69}$ Transparenzprobleme erkennen auch Vogenauer, Common Frame of Reference and UNIDROIT Principles of International Commercial Contracts, ERCL 2010, 183; Doralt, Strukturelle Schwächen in der Europäisierung des Privatrechts, RabelsZ 75 (2011), $270 \mathrm{f}$.

70 v. Bar/Clive (Fn. 1), S. 65-82.

${ }_{71}$ Mitteilung der Kommission an das Europäische Parlament und den Rat - Europäisches Vertragsrecht und Überarbeitung des gemeinschaftlichen Besitzstands - weiteres Vorgehen v. 11.10.2004, KOM (2004) 651 endg., 2.1.1, 3.1.3, Anhang I. 
Regeltextes und sollen Grundlage für eine einheitliche europäische Terminologie $\operatorname{sein}^{72}$. Die Definitionen der Unwirksamkeitsgrade haben wir bereits näher beleuchtet. Hier konnten wir feststellen, dass die Unwirksamkeitsgrade von den Verfassern nur ungenügend durchdrungen wurden und dass daher die gefundenen Definitionen nicht überzeugen ${ }^{73}$. Andere Definitionen sind verzichtbar. So wird etwa der Begriff contractual relationship zirkulär als „legal relationship resulting from a contract “ definiert $^{74}$. Wieder andere Definitionen verwirren ${ }^{75}$ :

„,Electronic' means relating to technology with electrical, digital, magnetic, wireless, optical, electromagnetic, or similar capabilities“.

Bei manchen Definitionen wird auf den Artikel, dem sie entnommen worden sind, verwiesen. Bei der Definition des Begriffs electronic fehlt ein solcher Hinweis. So bleibt zunächst unklar, in welchem Kontext etwa kabellose oder optische Vorrichtungen ausreichen sollen. Diese Frage stellt sich für die anschließende Definition der elektronischen Signatur ${ }^{76}$ :

„An ,electronic signature' means data in electronic form which are attached to, or logically associated with, other data and which serve as a method of authentication."

Hier wird der Leser auf Art. I.-1:108(3) verwiesen. Gemeint ist Art. I.1:107(3). Der Leser fragt sich, ob er die Definition des Begriffs electronic in die Definition der elektronischen Signatur hineinlesen darf. Der DCFR würde dann auch optische Signaturen kennen. Die Richtlinie 1999/93/EG nennt solche Signaturen nicht ${ }^{77}$. Die Frage klärt sich bei einer Lektüre von Art. I.-1:107(3) und (5): Die Verfasser wollen die Definition des Begriffs electronic in der Tat im Rahmen der Definition der elektronischen Signatur anwenden. Sie haben diese Definition nicht dem Unionsprivatrecht entnommen, sondern section 106(2) des amerikanischen Electronic Signatures in Global and National Commerce Act 2000 und gehen damit bewusst über Richtlinie 1999/93/EG hinaus. Mit der Frage, ob etwa eine optische Signatur im Anwendungsbereich des DCFR die Formzwecke erfüllen kann, wird der Leser allein gelassen. Eine offene Diskussion dieser Frage findet sich zu Art. I.-1:107 nicht. Der Leser erhält so den Eindruck, dass hier rein begrifflich gearbeitet wurde. Zudem stellt sich die Frage nach der Anwendbarkeit der Definition

72 v. Bar/Beale/Clive/Schulte-Nölke, Introduction, in: v. Bar/Clive (Fn. 1), S. 10.

73 Siehe oben den Text nach Fn. 41.

74 v. Bar/Clive (Fn. 1), S. 68.

75 v. Bar/Clive (Fn. 1), S. 70.

76 v. Bar/Clive (Fn. 1), S. 71.

77 RiL 1999/93/EG über gemeinschaftliche Rahmenbedingungen für elektronische Signaturen. 
von electronic auch in anderen Kontexten. Art. II.-3:105 behandelt den Vertragsschluss im elektronischen Geschäftsverkehr: Gibt es einen optischen Geschäftsverkehr?

Der Begriff fraudulent taucht in der Liste der Definitionen auf, wird aber nicht definiert, sondern nur in einem bestimmten Kontext erläutert ${ }^{78}$ :

„A misrepresentation is fraudulent if it is made with knowledge or belief that it is false and is intended to induce the recipient to make a mistake to the recipient's prejudice. A non-disclosure is fraudulent if it is intended to induce the person from whom the information is withheld to make a mistake to that person's prejudice."

Es werden also nur die Begriffe fraudulent misrepresentation und fraudulent non-disclosure erklärt. Doch taucht der Begriff fraudulent nicht nur in diesem Zusammenhang in Art. II.-7:205(2) auf, sondern in Art. IV.G.-3:105 f. auch in einem anderen Kontext. Die Definition einer presumption klingt zunächst unproblematisch ${ }^{79}$ :

„A ,presumption' means that the existence of a known fact or state of affairs allows the deduction that something else should be held true, until the contrary is demonstrated."

Die presumption ist mit der Vermutung des deutschen Rechts vergleichbar, die für das Eingreifen der Vermutungsfolge einen Vermutungstatbestand voraussetzt. Auf Art. II.-9:410 passt diese Definition nicht. Er enthält eine Liste von Klauseln, von denen vermutet wird, dass sie einen Verbraucher unangemessen benachteiligen. „A term [...] is presumed to be unfair [...],“ so beginnt dieser Artikel. Hier geht es nicht darum, auf Grundlage eines Erfahrungssatzes einen Vermutungstatbestand zu formulieren, wie es die Definition erfordert („allows the deduction“), sondern darum, den unbestimmten Rechtsbegriff des significant disadvantage in Art. II.-9:403 zu konkretisieren. Der Begriff presumption wird auch im Fall einer Beweislastumkehr verwandt, so in Art. IV.C.-8:109(3). Auch diese Vorschrift basiert nicht auf einem Erfahrungssatz, sondern reagiert auf eine andernfalls eintretende Beweisnot auf Seiten des Patienten.

Die Definitionen der Begriffe electronic, fraudulent und presumption sind also aus einem bestimmten Kontext herausgeschält und verallgemeinert worden, ohne zu prüfen, ob die jeweilige Verallgemeinerung auf alle Fälle passt, in denen der entsprechende Begriff verwendet wird. Damit werden die Begriffe schon im DCFR nicht einheitlich gebraucht. Das stellt den mit diesem Teil des DCFR verfolgten Zweck in Frage, nämlich dass die Definitionen

\footnotetext{
78 v. Bar/Clive (Fn. 1), S. 71.

79 v. Bar/Clive (Fn. 1), S. 77.
} 
Ausgangspunkt für die Entwicklung einer einheitlichen europäischen Terminologie sein sollen.

\section{Die vier Grundprinzipien}

Viele Regeln des zweiten Buches bieten einen weiten Auslegungsspielraum, weisen Anwendungslücken auf und bei zahlreichen Regeln besteht die Gefahr, dass Juristen mit ihrem nationalen Vorverständnis an sie herantreten und entsprechend lesen. Beispiele sind uns bereits begegnet: Art. II.-9:403405 ermöglichen keine Inhaltskontrolle von Klauseln in Verträgen zwischen Unternehmer und Nichtunternehmer, die keine Verbraucher sind ${ }^{80}$. Wie soll diese Schutzlücke geschlossen werden? Ist es ein Redaktionsversehen, dass es bei der Inhaltskontrolle im Unternehmerverkehr nach Art. II.-9:405 nicht darauf ankommt, dass die andere Partei benachteiligt wird ${ }^{81}$ ? Kann das neunte Kapitel des zweiten Buches analog auf juridical acts angewandt werden ${ }^{82}$ ? Wie sehen die „necessary adaptations“ bei Art. II.-7:101 aus ${ }^{83}$ ? Auf welche Weise müssen die identischen Kontrollmaßstäbe in Art. II.-9:403 und 404 unterschiedlich ausgelegt werden ${ }^{84}$ ? Können wir den Zurechnungsgedanken, der sich in Art. II.-9:102(4) findet, verallgemeinern und in Art. II.-4:102 und Art. II.8:101(3) hineinlesen ${ }^{85}$ ? Man könnte versuchen, die vier Grundprinzipien des DCFR zur Lösung dieser Probleme heranzuziehen. Dem Regeltext des DCFR sind insgesamt vier Prinzipien vorangestellt, die den Regeln zugrundeliegen sollen: Freiheit (freedom, im weiteren übersetzt als Privatautonomie), Rechtssicherheit (security), Gerechtigkeit (justice) und Effizienz $(\text { efficiency })^{86}$. Die Autoren des DCFR kamen mit der Formulierung solcher Prinzipien wiederum einem entsprechenden Auftrag nach ${ }^{87}$, formulieren aber auch ausdrücklich die Bedeutung solcher Prinzipien ${ }^{88}$ :

„Private law is one of those fields of law which are, or at least should be, based on and guided by deep-rooted principles."

80 Vgl. oben den Text nach Fn. 58.

81 Vgl. oben den Text nach Fn. 58.

82 Vgl. oben den Text nach Fn. 62.

83 Vgl. oben den Text nach Fn. 62.

84 Vgl. oben den Text zu Fn. 57.

85 Vgl. oben den Text zu Fn. 46.

86 v. Bar/Beale/Clive/Schulte-Nölke, Principles, in: v. Bar/Clive (Fn. 1), S. 37-63.

87 Vgl. Mitteilung (Fn. 71), 2.1.1, 3.1.3, Anhang I.

88 v. Bar/Beale/Clive/Schulte-Nölke, Introduction, in: v. Bar/Clive (Fn. 1), S. 5. 
Das Prinzip der Privatautonomie steht zu Recht am Anfang. Die unterschiedliche Bedeutung der Privatautonomie im Vertragsrecht, in außervertraglichen Schuldverhältnissen und im Sachenrecht arbeiten die vier Autoren der Prinzipien heraus. Aus deutscher Sicht bleiben die Ausführungen freilich zu sehr an der Oberfläche verhaften und sind zu wenig strukturiert. So werden Begriffe wie etwa Abschluss-, Inhalts- und Formfreiheit oder auch der formelle und der materielle Begriff der Vertragsfreiheit nur angedeutet. Interessant wäre zudem gewesen zu erfahren, wie sich die mitgliedstaatlichen Rechte der Privatautonomie nähern, wie sie sie herunterbrechen und welche Bedeutung ihr bei der Lösung einzelner Probleme zukommt. Die sehr allgemein gehaltenen Ausführungen bergen die Gefahr, dass Juristen aus unterschiedlichen Rechten glauben, ihr national geprägtes Vorverständnis der Privatautonomie im DCFR wiederzufinden, und dieses sodann in ihn hineinprojizieren. Anlass für tiefer gehende Darstellungen hätte es gegeben. So verstehen die Autoren die Anfechtungsmöglichkeit wegen Irrtums als Ausfluss der Vertragsfreiheit. Indes ist die Irrtumsanfechtung in Europa sehr unterschiedlich ausgestaltet, und man fragt sich, ob die Autoren aus dem Prinzip der Vertragsfreiheit eine bestimmte Ausgestaltung der Irrumsanfechtung herleiten.

Auch sind die Ausführungen an vielen Stellen so vereinfachend, dass sie nicht mehr richtig sind. So führen die Autoren zu der Frage aus, dass die Ausübung der Vertragsfreiheit voraussetzt, dass beide Parteien gleichermaßen über den Vertragsinhalt informiert sind ${ }^{89}$ :

„Modern law must also deal with lack of information as to the terms of the contract. The classical defences were developed at a time when most contracts were of a simple kind that the parties could understand readily. This too has changed, particularly with the development of longer-term (and therefore more complex) contracts and the use of standard terms."

Dass AGB bereits im 19. Jh. zum Massenphänomen wurden, ist lange bekannt. Auch komplexe Dauerschuldverhältnisse waren im 19. Jh. längst zu einem Massenphänomen geworden (Arbeitsverträge, Abzahlungsgeschäfte, Versicherungsverträge, Mietverträge). Auch das ist bekannt. Die Autoren gehen also noch über das inzwischen ebenfalls relativierte, für Deutschland sogar widerlegte ${ }^{90}$, Klischee hinaus, dass die Juristen des 19. Jh. keine Antworten auf die aus diesen Phänomenen resultierenden Probleme entwickelt hatten.

Schließlich überraschen einzelne Ausführungen. So führen die Autoren auch das Bereicherungsrecht auf die Privatautonomie zurück ${ }^{91}$ :

89 v. Bar/Beale/Clive/Schulte-Nölke, Principles, in: v. Bar/Clive (Fn. 1), S. 41.

90 Hierzu Hellwege, (Fn. 51), S. 21 ff.

91 v. Bar/Beale/Clive/Schulte-Nölke, Principles, in: v. Bar/Clive (Fn. 1), S. 43. 
„Again, in the law on unjustified enrichment the underlying principle is that people are free to hold what they have. An obligation to redress an enrichment is imposed only in carefully regulated circumstances.“

Einen Bereicherungsanspruch wegen Eingriffs, den auch der DCFR nach Art. VII.-4:101(c) kennt ${ }^{92}$, in ein Spannungsverhältnis zwischen der Freiheit des Schuldners, das durch Eingriff Erlangte behalten zu dürfen, und den Interessen des Verletzten, das durch Eingriff in seinen Zuweisungsgehalt Erlangte herausfordern zu dürfen, einzuordnen, ist aus deutscher Sicht zwar neu, aber auch wenig zielführend. Das Prinzip der Privatautonomie wird so mit wenig passenden Einzelheiten überfrachtet, und umgekehrt werden die Einzelregelungen in einen unpassenden Kontext gestellt, was wiederum ihre Auslegung negativ beeinflussen kann.

Den Begriff der security, hier übersetzt als Rechtssicherheit, verstehen die Autoren weit: Sie sei beeinträchtigt, wenn jemand in die Rechte eines anderen eingreife, wenn Gesetze unverständlich oder unzugänglich seien oder wenn ungewiss bleibe, ob der Vertragspartner erfülle und ob man gegen ihn effektive Mittel der Rechtsdurchsetzung habe. All dies wird nicht weiter vertieft, sondern es werden die Funktionen der (Rechts-)Sicherheit auf der Ebene des Vertragsrechts weiter aufgefächert. Hierher gehöre die Bindung an den Vertrag, der Grundsatz von Treu und Glauben, der Grundsatz, dass ein Vertrag im Zweifel so auszulegen sei, dass er wirksam sei, oder auch der Vertrauensschutz. Unter dem Stichwort des Vertrauensschutzes taucht wiederum die Irrtumsanfechtung auf. Sie dürfe nicht so ausgestaltet sein, dass der Erklärungsempfänger in seinem Vertrauen auf die Gültigkeit der Erklärung enttäuscht werde. Hier verweisen die Autoren nun zwar darauf, wie im Regeltext dieses Vertrauen geschützt wird. Aber eine eingehende Diskussion unterbleibt ebenso wie eine Verknüpfung mit den Ausführungen zur Irrtumsanfechtung unter dem Stichwort der Privatautonomie. Unter dem Begriff der Rechtssicherheit im Vertragsrecht ist weiterhin ein allgemeines Problem gefasst: Sollen Regeln einen Auslegungsspielraum eröffnen, der es ermöglicht, flexibel auf besondere Situationen zu reagieren, oder sollen sie bestimmt gefasst sein? Schließlich verweisen die Autoren noch auf die Regeln zur Verjährung und zu den persönlichen Sicherheiten, die ebenfalls der Rechtssicherheit im Vertragsrecht dienten. Dem Leser bleibt dabei unklar, warum die Verjährung nur im Vertragsrecht und nicht bei außervertraglichen Schuldverhältnissen Ausdruck der Rechtssicherheit sein soll und warum die Rechtssicherheit nur im Vertragsrecht durch die flexible Ausgestaltung von Regeln berührt sein soll.

$92 \mathrm{Vgl}$. zu den Einzelheiten und zum Verhältnis der bereicherungsrechtlichen Haftung zu Art. VI.-6:101(4): Monsen, DCFR and Restitution for Wrongs, 2010 ERPL $813 \mathrm{ff}$. 
Das dritte Prinzip ist die Gerechtigkeit, die die Autoren im Vertragsrecht auch durch den Gleichbehandlungsgrundsatz verwirklicht sehen. Vor dem Gesetz seien alle Bürger gleich. Die ungleiche Behandlung von Unternehmern und Verbrauchern verstehen die Autoren als Ausnahme. Sie sprechen sogar von einer großen Ausnahme ${ }^{93}$. Verbraucher und Unternehmer werden in der Tat ungleich behandelt. Dass dies aber vor dem Hintergrund des Gleichbehandlungsgrundsatzes ein bedeutendes Gerechtigkeitsproblem darstellt, war mir neu. Der Gleichheitsgrundsatz stehe auch hinter der synallagmatischen Verknüpfung von Leistung und Gegenleistung. Die Gleichbehandlung beider Parteien gebiete es, dass eine Partei nur dann erfüllen müsse, wenn auch die andere Partei erfülle. Weiter sei es ein Gebot der Gerechtigkeit zu unterbinden, dass Parteien aus ihrem eigenen rechtswidrigen Verhalten einen Vorteil ziehen könnten. Auch sei der Verbraucherschutz ein Ausdruck des Gerechtigkeitsprinzips. Damit ist nach Ansicht der Verfasser der Verbraucherschutz ein Gebot der Gerechtigkeit (Schutz des Schwächeren), und er steht zugleich in Widerspruch zur Gerechtigkeit (Ungleichbehandlung mit Unternehmern). Das hätte man sicher näher erläutern dürfen. Nur für das Sachenrecht formulieren die Autoren schließlich noch Folgendes: Das Gerechtigkeitsprinzip sei im DCFR verwirklicht, weil dessen Regeln einen gerechten Ausgleich zwischen den in Frage stehenden Parteiinteressen darstellen ${ }^{94}$. Warum dieser Gedanke gerade für das Sachenrecht so betont wird, bleibt unklar.

Das vierte Prinzip ist das der Effizienz (efficiency). Die Formfreiheit, die nicht bei Diskussion der Privatautonomie genannt wurde, wird als Ausdruck der Effizienz verstanden. Dass die Vertragsanfechtung so wie in Deutschland durch gestaltende Erklärung bewirkt werden kann und nicht wie in anderen europäischen Rechten ein entsprechendes Urteil erwirkt werden muss, fördere ebenfalls die Effizienz. Auch die Nichtanerkennung der considerationLehre des englischen Rechts und die Bindung an einseitige Versprechen in Art. II.-1:103(2) wird überraschenderweise nicht auf die Privatautonomie der Parteien bzw. des Versprechenden zurückgeführt, sondern mit Effizienzgedanken begründet. Weiterhin sei die Existenz des dispositiven Rechts insgesamt der Effizienz geschuldet: Die Parteien sollen auf das dispositive Recht zurückgreifen können in der Gewissheit, dass dieses einen fairen Ausgleich ihrer Interessen enthalte. Daher habe der DCFR auch auf die Einführung sogenannter penalty default rules verzichtet.

In der Interim Outline Edition fanden sich noch weitere Prinzipien, so etwa die Bewahrung der kulturellen und sprachlichen Vielfalt (preservation of cultural and linguistic plurality). In der vollständigen Ausgabe hat dieses

93 v. Bar/Beale/Clive/Schulte-Nölke, Principles, in: v. Bar/Clive (Fn. 1), S. 53.

94 v. Bar/Beale/Clive/Schulte-Nölke, Principles, in: v. Bar/Clive (Fn. 1), S. 59. 
Prinzip seine eigenständige Bedeutung verloren. Freilich meinen die Autoren der Einleitung, dass die nun nicht mehr ausdrücklich genannten Prinzipien ihm noch inne wohnen ${ }^{95}$. Als Beispiel für die Bewahrung der kulturellen und sprachlichen Vielfalt verweisen sie etwa auf Art. II.-1:104(2) ${ }^{96}$ :

„The parties are bound by a usage which would be considered generally applicable by persons in the same situation as the parties, except where the application of such usage would be unreasonable."

Die Autoren der Einleitung führen aus, dass diese Regel auch die kulturelle Vielfalt bewahre, weil von ihr lokal begrenzte Gewohnheiten erfasst seien ${ }^{97}$. Aus deutscher Sicht liegen solche Ausführungen neben der Sache. Lokale Handelsbräuche sind nicht deshalb zu beachten, weil sie Ausfluss einer lokal beschränkten Kultur sind.

In der Gesamtschau überzeugt dieser Abschnitt nicht ${ }^{98}$ : Die Ausführungen zu den Grundwertungen, die hinter den Regeln des DCFR stehen, wirken, als seien sie das Produkt eines brain-stormings. Als Folge wird unter den vier Prinzipien vollkommen Unzusammenhängendes vereint und einfach nebeneinander gestellt, Ausprägungen der Prinzipien auf unterschiedlichen Abstraktionsebenen ohne Unterschied aufgezählt, das Zusammenspiel der Prinzipien und der Widerstreit zwischen ihnen nur unvollkommen beleuchtet. Auch überzeugen die Zuordnungen einfacher Regeln zu einem Prinzip oft nicht bzw. die einfachen Regeln wirken dadurch überfrachtet. Insgesamt sind die Ausführungen ohne wissenschaftlichen und praktischen Wert. Ja, sie sind umgekehrt sogar schädlich. Die eingangs gestellte Frage lautete, ob die Prinzipien nutzbar gemacht werden können, um die zahlreichen Auslegungsprobleme zu lösen. Ich denke nicht ${ }^{99}$ : So hilft die zweifelhafte Herleitung der Bindungswirkung einseitiger Versprechen aus Effizienzgedanken kaum bei Beantwortung der Frage, ob und wie das neunte Kapitel auf solche einseitigen Versprechen angewendet werden kann. Und dass man die Berücksichtigung lokaler Gewohnheiten und Bräuche auf die Bewahrung der kulturellen und sprachlichen Vielfalt zurückführt, wird die Auslegung des entsprechenden Artikels eher hindern.

95 v. Bar/Beale/Clive/Schulte-Nölke, Introduction, in: v. Bar/Clive (Fn. 1), S. 8-10.

96 Vgl. zu Art. II.-1:104(2) Eidenmüller/Faust/Grigoleit/Jansen/Wagner/Zimmermann, JZ 2008, 538.

${ }_{97}$ v. Bar/Beale/Clive/Schulte-Nölke, Introduction, in: v. Bar/Clive (Fn. 1), S. 9 Fn. 25.

98 Vgl. auch die Kritik zu den Prinzipien der Interim Outline Edition Eidenmüller/ Faust/Grigoleit/Jansen/Wagner/Zimmermann, JZ 2008, 535.

99 Mit Blick auf die Auslegung von Art. II.-9:405 bereits Mangeri, ERCL 2011, 223. 


\section{Die möglichen Funktionen des Draft Common Frame of Reference}

Vorstehend haben wir den acquis communautaire und die Vereinheitlichungsprojekte in den Blick genommen und gefragt, ob der DCFR Fortschritte bringt, Abweichungen von Vorgängertexten genügend begründet und die bekannten Defizite in ihnen abzuhelfen vermag ${ }^{100}$. Wir haben auch das deutsche Recht als Folie genutzt ${ }^{101}$ und erörtert, ob der DCFR aus seiner Sicht vorzugswürdig oder auch nur akzeptabel ist. Und wir haben den DCFR isoliert bewertet und die Regeln und Begriffe auf ihre Kohärenz überprüft. All das sind mögliche Wege, den DCFR zu würdigen. Man kann auch mögliche Wechselwirkungen zwischen nationalem Recht und DCFR untersuchen ${ }^{102}$. Und man kann die Frage aufwerfen, ob die vollständige Ausgabe des DCFR geeignet ist, die mit ihr verknüpften Funktionen zu erfüllen. Dies soll, wiederum mit Blick auf das zweite Buch, nachfolgend versucht werden. Freilich ist noch offen, welche Funktionen der DCFR erfüllen wird ${ }^{103}$ : Wird es ein optionales Vertragsrecht geben, dessen Vorentwurf der DCFR ist? Wird der DCFR der erste Schritt hin zu einer europäischen Kodifikation sein? Wird der DCFR eine tool box bleiben, an der sich der europäische und die nationalen Gesetzgeber orientieren werden? Oder handelt es sich um ein rechtsvergleichendes Referenzwerk?

Die sechs Bände der vollständigen Ausgabe des DCFR stellen den akademischen DCFR dar. Sie seien ein rechtsvergleichendes Referenzwerk: So sieht Schulte-Nölke den Zustand der Rechtsvergleichung in Europa kritisch. Sie habe selten alle europäischen Rechte im Blick. Es sei ein „Desiderat, Methoden und Darstellungsweisen zu finden, mit denen, viel genauer als bisher, eine Landkarte der europäischen Rechtsordnungen gezeichnet werden kann“104. Ein solches Werk dürfe nicht der Ordnung des BGB folgen. Eine eigene Ordnung müsse geschaffen werden. Eine solche Ordnung stellten die

100 So auch der Ansatz von Zimmermann, Textstufen in der modernen Entwicklung des europäischen Privatrechts, EuZW 2009, 319 ff. und der Beiträge in: Schulze (Fn. 21).

101 So auch der Ansatz der Beiträge in: Antoniolli/Fiorentini (Fn. 55).

102 So eine von Vogenauer/Dannemann initiierte Gruppe für das deutsche und englische Recht. Vgl. http://cfr.iuscomp.org/ (zuletzt besucht am 23.3.2011).

$103 \mathrm{Vgl}$. die detaillierte Diskussion der verschiedenen Optionen bei Max Planck Institute for Comparative and International Private Law. Policy Options for Progress Towards a European Contract Law. Comments on the issues raised in the Green Paper from the Commission of 1 July 2010, COM(2010) 348 final, RabelsZ 75 (2011), $371 \mathrm{ff}$.

104 Schulte-Nölke, Arbeiten an einem europäischen Vertragsrecht, NJW 2009, $2161 \mathrm{f}$. 
Regeln des DCFR, und der DCFR solle die identifizierte Lücke schließen. Das im DCFR ausgebreitete Regelwerk solle das Gerüst sein, anhand dessen die rechtsvergleichende Darstellung erfolgen könne. Das Regelwerk sei dabei seinerseits rechtsvergleichend erarbeitet worden und enthalte Regeln, die den europäischen Rechten gemeinsam (common rule-approach) oder die vorzugswürdig (best rule-approach) seien.

Diesen Ansprüchen wird das zweite Buch des DCFR nicht gerecht ${ }^{105}$ : So wird nicht überall die Rechtslage in ganz Europa dargestellt. Wir finden etwa in den notes zu Art. II.-3:401 nur Hinweise auf das Unionsprivatrecht sowie das estnische, slowenische, slowakische, britische und bulgarische Recht. Das ist keine Landkarte aller europäischen Rechte. Zudem begnügen sich die Verfasser oft mit dem Hinweis, dass ein bestimmtes Recht einer bestimmten Lösung folge, ohne dabei die gesetzlichen Bestimmungen dieses Rechts zu nennen, so in note $3 \mathrm{zu}$ Art. II.-9:405 ${ }^{106}$. Solch pauschale Hinweise sind ohne Wert. Manchmal fehlt sogar jede Bezugnahme auf irgendwelche Rechte. So lesen wir in note 14 wieder zu II.-9:405 ${ }^{107}$ :

„Furthermore, the process of reviewing incorporation and interpretation often represents a hidden form of content review, in which not only formal aspects are examined. Thus, in a number of Member States the incorporation of standard terms does not merely depend upon whether the other party has had the opportunity of becoming acquainted with the contractual terms [...]. Rather, in some of the Member States the content of the clause (and thus its fairness) are considered as well when deciding whether or not a term has been incorporated into the contract."

Der Leser erfährt nicht, welche Rechte gemeint sind. Er bekommt so das Gefühl, die Verfasser haben hier nur ein verbreitetes Klischee übernommen, ohne es rechtsvergleichend zu überprüfen.

Zudem muss eine Karte nicht nur vollständig sein, damit sie von Wert ist, sie darf zudem keine Fehler enthalten. Auch insoweit muss sich das zweite Buch des DCFR Kritik gefallen lassen: So heißt es in note 9 zu Art. II.9:407, dass $\$ 305$ Abs. 2 BGB auch im Unternehmerverkehr zur Anwendung komme. Und in note $3 \mathrm{zu}$ Art. II.-9:410 wird behauptet, $\ 310$ Abs. 1 S. 2 BGB führe dazu, dass den Klauselkatalogen der $\$ \$ 308 \mathrm{f}$. BGB auch im Unternehmerverkehr eine starke Indizwirkung zukomme. Auch das ist nicht richtig. \$310 Abs. 1 S. 1 BGB bestimmt, dass $\mathbb{\int} 308 \mathrm{f}$. BGB im Unternehmerverkehr nicht zur Anwendung kommen. $\$ 310$ Abs. 1 S. 2 BGB besagt nur, dass eine Klausel, die nach $\mathbb{\$} \$ 308 \mathrm{f}$. BGB unwirksam wäre, nach $\$ 307$ Abs. 1

105 Kritisch auch Jansen/Zimmermann, Was ist und Wozu der DCFR?, NJW 2009, $3401 \mathrm{ff}$.

106 v. Bar/Clive (Fn. 1), Note 3 zu Art. II.-9:405 (S. 643).

107 v. Bar/Clive (Fn. 1), Note 14 zu Art. II.-9:405 (S. 645). 
und 2 BGB unwirksam sein kann. Die Indizwirkung wird nicht durch $₫ 310$ Abs. 1 S. 2 BGB angeordnet, sondern ist Ergebnis einer (umstrittenen) Rechtsprechung ${ }^{108}$. In note 8 zu Art. II.-9:408 heißt es, eine geltungserhaltende Reduktion sei in Österreich und Großbritannien umstritten. Dagegen sei es in Deutschland „established in legal literature that a reduction is inadmissable“. Freilich ist das Verbot der geltungserhaltenden Reduktion auch in der deutschen Literatur umstritten ${ }^{109}$. Nun läuft man in der Rechtsvergleichung immer das Risiko, ein fremdes Recht misszuverstehen. Aber in einem Werk, das sich zur Aufgabe setzt, eine Landkarte der europäischen Rechte zu zeichnen, sind solche Fehler ärgerlich, vor allem auch deshalb, weil es sich nicht um das Werk eines einzelnen Forschers handelt, sondern weil an dem Forschernetzwerk Juristen aus allen Mitgliedstaaten beteiligt waren. Das Risiko von Fehlern hätte minimiert werden können und müssen. Und entdeckt man bei der Darstellung des eigenen Rechts nicht nur vereinzelt Fehler, so wird man sich nicht auf die Darstellung der fremden Rechte verlassen wollen.

Zudem beschränken sich die Verfasser oft auf einen Vergleich bloßer Normtexte. Als Folge finden sie nicht immer die funktionalen Äquivalente, und die Darstellung wird unvollständig: note 6 zu Art. II.-9:402 klärt auf, dass im deutschen Recht eine intransparente Klausel nach $\$ 305$ c Abs. 2 BGB contra proferentem ausgelegt und dass die Intransparenz nach $₫ 307$ Abs. 1 S.2 BGB auch im Rahmen der Inhaltskontrolle berücksichtigt werden könne. Zum lettischen Recht heißt es sodann in note $9^{110}$ :

„In Latvia, although the legal consequences are not regulated in the ConsProtA, general norms of civil law could be nevertheless applied, particularly CC art. 1506 stating that absolutely disreputable and unintelligible and also contradictory terms are not to be interpreted at all, but deemed null and void.“

Aber gleiches gilt auch nach deutschem Recht. Zwar finden wir hierzu keine ausdrückliche Regel im BGB, aber eine perplexe Klausel ist nach den Grundsätzen der Rechtsgeschäftslehre nichtig ${ }^{111}$. Weitere Beispiele könnten ergänzt werden. Die Verfasser des DCFR beklagen also den Zustand der Rechtsvergleichung in Europa, als nicht alle Rechte berücksichtigt werden. Hier wollen sie Abhilfe schaffen. Dies geschieht um den Preis, dass die einzelnen Rechte fehlerhaft und nur noch fragmentarisch dargestellt werden. Das alles schränkt die Brauchbarkeit als rechtsvergleichendes Referenzwerk ein.

108 Vgl. nur Staudinger/Coester, Neubearbeitung 2006, \307 Rn. 14.

109 Vgl. nur MK-BGB/Basedow, 5. Aufl. 2007, \306 Rn. $12 \mathrm{ff.}$

110 v. Bar/Clive (Fn. 1), Note 9 zu Art. II.-9:402 (S. 633).

111 Vgl. Medicus (Fn. 29), Rn. 759. 
Die vollständige Ausgabe des DCFR soll nicht ein akademischer Text bleiben, sondern wurde auch als Vorentwurf zu einem politischen CFR verfasst $^{112}$. Offen ist freilich noch, welche Funktion der politische CFR erfüllen soll. Viel spricht dafür, dass ein optionales Instrument in Kraft gesetzt wird $^{113}$. Neben dem optionalen Instrument wird vorgeschlagen, der DCFR könne als akademischer Entwurf oder nach Überarbeitung als politischer CFR Inspirationsquelle für den europäischen und die nationalen Gesetzgeber sein ${ }^{114}$, beide könnten mithin eine tool box sein. Auch könnten DCFR und CFR „dem EuGH als Anhaltspunkt bei der Auslegung des Gemeinschaftsprivatrechts und den nationalen Gerichten bei der gemeinschaftsrechtskonformen Auslegung des nationalen Rechts dienen" ${ }^{\text {"115. }}$. Und schließlich habe der DCFR die Qualität, um unmittelbar Vorentwurf für ein europäisches Zivilgesetzbuch zu sein ${ }^{116}$. Optionales Instrument, tool box, Auslegungshilfe oder europäisches Zivilgesetzbuch, der DCFR hat nur dann das Zeug dazu, jeweils als Vorentwurf hierfür zu fungieren, wenn die Regeln überzeugen. Dass dies für das zweite Buch vor allem dort nicht der Fall ist, wo die Verfasser Neuland betreten haben, wurde oben dargetan. An dieser Stelle möchte ich noch zwei Punkte ergänzen:

Der erste Punkt bezieht sich auf das methodische Vorgehen der Verfasser. Sie hätten rechtsvergleichend gearbeitet und versucht, eine gemeinsame Regel bzw. die für ein gemeineuropäisches Privatrecht beste Regel herauszudestillieren. Freilich verwundert dann, dass die Autoren der Einleitung auf Zeitgründe verweisen, warum die rechtsvergleichenden Hinweise zum Schenkungsrecht nicht mehr angefertigt wurden ${ }^{117}$. Man würde doch denken, dass das rechtsvergleichende Material bereits vorhanden und durchdrungen

112 Vgl. Briggs, [2009] LMCLQ 158; Doralt, Rote Karte oder grünes Licht für den Blue Button?, AcP 211 (2011), 3 ff.; ders., RabelsZ 75 (2011), 266 f.

113 Vgl. nur Reding, Warum Europa ein optionales Europäisches Vertragsrecht benötigt, ZEuP 19 (2011), 1 ff. Zu den verschiedenen Möglichkeiten des Anwendungsbereichs des optionalen Instruments vgl. nur Doralt, AcP 211 (2011), 6 ff.; Max Planck Institute for Comparative and International Private Law, RabelsZ 75 (2011), $420 \mathrm{ff}$.; Schulte-Nölke, How to realise the ,Blue Button'?, in: Schulze/Schulte-Nölke (Hg.), European Private Law - Current Status and Perspectives, 2011, S. 89 ff.; Augenhofer, A European Civil Law - for Whom and What Should it Include?, ERCL 2011, $208 \mathrm{ff}$.

114 v. Bar/Beale/Clive/Schulte-Nölke, Introduction, in: v. Bar/Clive (Fn. 1), S. 19.

115 Leible, Europäisches Privatrecht am Scheideweg, NJW 2008, 2561.

116 Dafür Hesselink, (2009) 83 Tulane LR 923. Reding bezeichnet den DCFR als „our embryo for a European civil code“, zitiert bei Lord Mance, ZEuP 18 (2010), 457. Einschränkend $v$. Bar, A Common Frame of Reference for European Private Law, (2008) 23 Tulane European and Civil Law Forum, 37 ff., 40. Dagegen Leible, NJW 2008, 2562. Zum ganzen Jansen/Zimmermann, NJW 2009, $3401 \mathrm{ff.}$

117 v. Bar/Beale/Clive/Schulte-Nölke, Introduction, in: v. Bar/Clive (Fn. 1), S. 2. 
ist, bevor die Regel formuliert wird, und dass als Folge auch die rechtsvergleichenden notes zeitgleich mit dem Regeltext verfasst wurden oder werden konnten ${ }^{118}$.

Wurde der Regeltext rechtsvergleichend erarbeitet, so müssen die Verfasser nach den anerkannten Methoden der Rechtsvergleichung vorgegangen sein. Dies ist, zumindest in Deutschland, die funktionale Methode. Nun führen die Verfasser in ihren rechtsvergleichenden notes in der Tat funktionale Äquivalente an. Aber sie zitieren vor allem gesetzliche Normen. Die funktionale Methode geht indes über einen reinen Normenvergleich hinaus, indem sie auch die Rechtsprechung und die Literatur mit einbezieht. Beides wird in den rechtsvergleichenden notes vernachlässigt. Als Folge bleiben die Verfasser oft am bloßen Wortlaut der Norm hängen. So verweisen die notes zu Art. II.9:403 darauf, dass viele Mitgliedstaaten Klauseln in Verbraucherverträgen als missbräuchlich erachten, wenn sie zu einem erbeblichen Ungleichgewicht führten, andere stellten dagegen auf eine unangemessene Benachteiligung ab, wobei einige, aber nicht alle als zusätzliche Voraussetzung verlangten, dass diese Benachteiligung bzw. dieses Ungleichgewicht gegen guten Glauben verstoßen müsse. Und im litauischen Recht sei nicht der gute Glaube, sondern der gute Wille zusätzlicher Kontrollmaßstab. Die entscheidende Frage, wie diese Kontrollmaßstäbe angewandt werden, wird nicht gestellt. Trotz einer identischen Fassung des Normtextes können sich die Ergebnisse in der Praxis ja unterscheiden. Und die unterschiedliche Fassung einer Norm kann in der Praxis bedeutungslos bleiben. Auch hier erwecken die Verfasser den Eindruck, als hätten sie bloß begrifflich gearbeitet.

Rechtsvergleichung darf nicht dabei stehen bleiben, Unterschiede und Ähnlichkeiten zu identifizieren. Sie muss Unterschiede erklären. Und sie muss herausarbeiten, ob Ähnlichkeiten nur oberflächlich existieren. Diesen Anspruch kann die Rechtsvergleichung nur erfüllen, wenn sie sich weiteren Methoden öffnet: Sie muss die unterschiedlichen rechtlichen Traditionen, die Rechtsgeschichte, unterschiedliche vertragsrechtstheoretische Ansätze, die unterschiedlichen Wertungen, die hinter vermeintlich identischen Normen stehen, und vieles mehr berücksichtigen. Nun konnte es von den Verfassern nicht erwartet werden, dass sie dies in der Kürze der Zeit und bei der Masse des zu bewältigenden Stoffes leisten. Will der akademische DCFR dem Forscher nur als Ausgangspunkt für vergleichende Forschung dienen, so muss der Forscher diese Aufgabe nun selbst angehen. Indes, eine vorzugswürdige Regel (best rule-approach), die als Vorentwurf eines wie auch immer gearteten Gesetzgebungsakts herangezogen werden kann, hätten auch die Verfasser des DCFR nur erarbeiten können, wenn sie über den Normtext hinaus-

118 Vgl. auch Vogenaner, ERCL 2010, 183. 
gehen. Gleiches gilt dort, wo die Verfasser sich auf eine Fortentwicklung des acquis beschränken: note $1 \mathrm{zu}$ Art. II.-9:405, der die Generalklausel zur Inhaltskontrolle von AGB im Unternehmerverkehr enthält, erklärt, dass die Formulierung des Kontrollmaßstabes Art. 3(3) der Richtlinie 2000/35/EG EG $^{119}$ entstammt. Ob Art. II.-9:405 und dieser Art. 3(3) den gleichen Schutzzweck haben, ob die Formulierung des Art.3(3) überhaupt passt, diskutieren die Verfasser nicht. Auch hier scheinen die Verfasser allein Wert auf eine vordergründig einheitliche Terminologie gelegt zu haben.

Der zweite Punkt bezieht sich auf die Präsentation der Regeln. Obwohl klar war, dass der DCFR Vorentwurf für einen politischen CFR sein wird, orientierten sich die Verfasser auch hier an vielfachen Vorbildern, so den $R e-$ statements und den PECL. Aber diese Vorbilder dienten einer anderen Funktion. Sie sollten durch ihre Qualität und die wissenschaftliche Autorität ihrer Verfasser wirken. Der DCFR ist dagegen möglicherweise Vorentwurf für einen Gesetzgebungsakt. Diese Funktionsverschiebung hätte eine andere Präsentation der Regeln notwendig gemacht ${ }^{120}$. Wir finden nach dem Regeltext erläuternde comments und rechtsvergleichende notes ${ }^{121}$ :

„The comments explain the objective of the particular model rule, place it in the context of the DCFR as a whole and, where appropriate, provide information about its origins and discuss possible alternative solutions. [...] The notes give the present legal position in the Member States and in Community law (where available).“

Ursprung der Regelung und alternative Lösungsansätze finden sich nur „where appropriate“. Das ist im zweiten Buch äußerst selten. Als Folge wird der Regeltext nirgends begründet. Es handelt sich, aus deutscher Sicht, um keine Gesetzesbegründung, sondern um eine Kommentierung. Das ist unbefriedigend $^{122}$ : Mit einer Regelbegründung hätten die methodischen Bedenken ausgeräumt werden können. Zudem fehlt die Begründung für eine Auslegung: Viele Regeln bieten ja einen weiten Auslegungsspielraum, weisen Anwendungslücken auf und bei zahlreichen Regeln besteht die Gefahr, dass Juristen mit ihrem nationalen Vorverständnis an sie herantreten und entsprechend lesen. Beispiele sind uns bereits begegnet. Die daraus resultierenden Fragen können nur beantwortet werden, wenn die Ursprünge der Regeln, alternative Lösungsansätze, die hinter ihnen stehenden Wertungen sehr viel ausführlicher bzw. überhaupt diskutiert worden wären. Eine bloße Erläuterung, wie die Regel angewendet wird, ist hier ohne jeden Wert. Eine solche Begründung wäre auch nötig gewesen, um einschätzen zu können, wann ein nationales

119 RiL 2000/35/EG zur Bekämpfung von Zahlungsverzug im Geschäftsverkehr.

120 Kritisch auch Jansen/Zimmermann, NJW 2009, 3406.

121 v. Bar/Beale/Clive/Schulte-Nölke, Introduction, in: v. Bar/Clive (Fn. 1), S. 3.

122 Kritisch auch Whittaker (Fn. 25), S. 3 f. 
Vorverständnis fehlleitet. Und schließlich wäre eine Begründung dort notwendig gewesen, wo der Regeltext über den acquis communautaire hinausgeht oder vom acquis commun oder von Vorgängertexten des europäischen Privatrechts abweicht. Freilich richtet sich diese Kritik nicht gegen den DCFR als akademisches Werk. Die Kritik ist nur berechtigt, als der DCFR auch als Vorentwurf für einen politischen CFR fungieren soll. Dieser bedarf einer ausführlichen Begründung. Aber wer sollte diese Begründung liefern, wenn nicht die Verfasser des akademischen DCFR?

\section{$X V$. Schluss}

Eine umfassende Würdigung des zweiten Buches des DCFR war in diesem Beitrag nicht möglich. Doch deuten die angeführten Beispiele auf immer wieder auftretende Defizite hin: (1) Die Verfasser verwenden Begriffe uneinheitlich, und das obwohl sie gerade eine im Vergleich zu den PECL klarere Begrifflichkeit anstrebten. (2) Zudem hätten sich die Verfasser öfters die Frage nach der Regelungsbedürftigkeit eines Problems stellen sollen ${ }^{123}$. Dass es in den Mitgliedstaaten zu gemischten Verträgen kaum gesetzliche Regelungen gibt, spricht etwa dafür, dass es einer ausdrücklichen Regelung nicht bedarf. (3) Dort, wo der DCFR vom acquis communautaire oder den PECL abweicht oder darüber hinausgeht, überzeugen die gefundenen Regeln aus unterschiedlichen Gründen nicht immer: (a) Einige Regeln, etwa die zu den vorvertraglichen Pflichten, müssen sich einer rechtspolitischen Debatte stellen. (b) Andere enthalten handwerkliche Fehler, so die lückenhaften Generalklauseln zur Inhaltskontrolle. (c) Bei wieder anderen fragt man sich, warum die Verfasser sie nicht an Beispielen des täglichen Lebens durchgespielt haben, so bei Art. II.-5:202. (d) Und oftmals wurden verschiedene Regelungsbereiche nur ungenügend aufeinander abgestimmt, so die vorvertraglichen Informationspflichten auf die Einbeziehungsvoraussetzungen und die Auslegungsregeln. (4) Auch ist das zweite Buch in der vorgelegten Fassung nicht geeignet, die mit dem DCFR verbundenen Funktionen zu erfüllen. (5) Und schließlich deuten die rechtsvergleichenden notes darauf hin, dass die Verfasser nicht immer nach den anerkannten Methoden der Rechtsvergleichung gearbeitet haben.

Die Verfasser haben damit allenfalls einen Diskussionsentwurf vorgelegt, der sich zu den bereits bestehenden Modellregeln hinzugesellt. Die Gründe dafür, dass sie ihre Ziele verfehlt haben, liegen auf der Hand: Nicht alle Regelungsbereiche, die vom zweiten Buch erfasst sind, sind gleichermaßen rechts-

123 Vgl. zum übertriebenen Vollständigkeitsstreben des DCFR kritisch auch Vogenauer, ERCL 2010, 164 ff, 166: „exaggerated striving for completeness“. 
vergleichend aufgearbeitet. Zudem steht noch eine kritische Durchdringung des gemeinschaftlichen Besitzstandes aus ${ }^{124}$. Es fehlten mithin die Vorarbeiten, auf welche die Verfasser hätten zurückgreifen können. Diese Ausgangslage erlaubte es ihnen nicht, in der gewährten kurzen Zeit einen ausgereiften Text vorzulegen.

Vor dem nächsten Schritt hin zu einem politischen CFR muss daher eine Auszeit genommen werden: Die verschiedenen Regelwerke müssen miteinander verglichen werden, die mitgliedstaatlichen Wissenschaften müssen die Gelegenheit erhalten, diese aus der Perspektive ihres jeweiligen Rechts kritisch zu beleuchten, und der DCFR muss auf seine Kohärenz hin weiter untersucht werden.

Dennoch schreitet die Entwicklung (zu) schnell weiter voran: Im April 2010 setzte die Kommission eine Expertengruppe zur Überarbeitung des DCFR $e^{125}{ }^{125}$. Ihre Zusammensetzung ${ }^{126}$ ist auf berechtigte Kritik gestoßen ${ }^{127}$. Inzwischen hat die Expertengruppe ihre Ergebnisse vorgelegt ${ }^{128}$. Doch sind die Mängel des zweiten Buches des DCFR zu zahlreich und zu grundlegend, als dass die Expertengruppe sie in der zur Verfügung stehenden Zeit hätte abstellen können: So vereinigt etwa Art.40(1) dieser Feasibility Study for a Future Instrument in European Contract Law anders als Art. II.-5:201(1) nicht mehr die beiden Widerrufsrechte bei Haustürgeschäften und bei Fernabsatzverträgen. Auch ist das Widerrufsrecht bei sogenannten off-premise contracts (zuvor Haustürgeschäfte) nach Art. 40(1)(b) nicht mehr so weit gefasst, dass die oben aufgezeigten Anwendungsprobleme bestehen ${ }^{129}$, wenn man die Definitionen der Begriffe off-premise contract und business premises in Art. 2 mitberücksichtigt. Aber nun besteht bei Zeitungsständen, von denen aus Passanten aggressiv angesprochen werden, um ein Abonnement abzuschließen, kein Widerrufsrecht mehr, soweit man, was naheliegt, die entsprechenden Zeitungsstände als „movable retail premises where a business carries on activity on a usual basis" versteht, und das obwohl die Autoren des DCFR hier noch ein Widerrufsrecht gewähren wollten ${ }^{130}$. Der gleichen Kritik ist der ähnlich

124 Statt aller Augenhofer, ERCL 2011, 207 f.; Max Planck Institute for Comparative and International Private Law, RabelsZ 75 (2011), 399.

125 Beschluss 2010/233/EU der Kommission zur Einsetzung einer Expertengruppe für einen gemeinsamen Referenzrahmen im Bereich des europäischen Vertragsrechts.

126 Eine Liste mit den Mitgliedern der Gruppe ist abgedruckt in ZEuP 18 (2010), 955.

127 Vgl. nur Doralt, AcP 211 (2011), 5 f.; ders., RabelsZ 75 (2011), 277 ff.

128 Abrufbar unter: http://ec.europa.eu/justice/policies/consumer/docs/explana tory_note_results_feasibility_study_05_2011_en.pdf (zuletzt besucht am 21.6.2011).

${ }_{129}$ Siehe oben den Text zu und nach Fn. 34.

130 Siehe oben den Text zu Fn. 37. 
formulierte Art. 9 der European Parliament legislative resolution on the proposal for a directive of the European Parliament and of the Council on consumer rights vom 23.6.2011 ausgesetzt ${ }^{131}$. Und die Vorschriften der Feasibility Study zur Inhaltskontrolle in Art. 77 ff. weichen in so erheblichem Umfang von Art. II.-9:401 ff. ab, dass man nicht glauben möchte, der DCFR habe hier überhaupt als Vorentwurf gedient, ohne dass die Art. 77 ff. dabei im Einzelnen weniger problematisch sind als die Regeln des DCFR.

131 Abrufbar unter: http://www.europarl.europa.eu/document/activities/cont/201 106/20110624ATT22578/20110624ATT22578EN.pdf (zuletzt besucht am 25.6.2011). 